\title{
A Reduced Basis Hybrid Method for the Coupling of Parametrized Domains Represented by Fluidic Networks
}

\author{
Laura Iapichino ${ }^{\star}$, Alfio Quarteroni*, ${ }^{\star}$, Gianluigi Rozza* \\ ${ }^{\star}$ Chair of Modelling and Scientific Computing (CMCS) Mathematics Institute of \\ Computational Science and Engineering (MATHICSE), École Polytechnique \\ Fédérale de Lausanne, EPFL, Station 8, CH-1015 Lausanne, Switzerland. \\ $\S M O X-$ Modellistica e Calcolo Scientifico, Dipartimento di Matematica \\ "F. Brioschi" Politecnico di Milano, via Bonardi 9, 20133 Milano, Italy
}

\begin{abstract}
In this paper we propose a reduced basis hybrid method (RBHM) for the approximation of partial differential equations in domains represented by complex networks where topological features are recurrent. The RBHM is applied to Stokes equations in domains which are decomposable into smaller similar blocks that are properly coupled.

The RBHM is built upon the reduced basis element method (RBEM) and it takes advantage from both the reduced basis methods (RB) and the domain decomposition method. We move from the consideration that the blocks composing the computational domain are topologically similar to a few reference shapes. On the latter, representative solutions, corresponding to the same governing partial differential equations, are computed for different values of some parameters of interest, representing, for example, the deformation of the blocks. A generalized transfinite mapping is used in order to produce a global map from the reference shapes of each block to any deformed configuration.

The desired solution on the given original computational domain is recovered as projection of the previously precomputed solutions and then glued across subdomain interfaces by suitable coupling conditions.

The geometrical parametrization of the domain, by transfinite mapping, induces non-affine parameter dependence: an empirical interpolation technique is used to recover an approximate affine parameter dependence and a subsequent offline/online decomposition of the reduced basis procedure. This computational decomposition yields a considerable reduction of the problem
\end{abstract}


complexity. Results computed on some combinations of 2D and 3D geometries representing cardiovascular networks show the advantage of the method in terms of reduced computational costs and the quality of the coupling to guarantee continuity of both stresses, pressure and velocity at subdomain interfaces.

Keywords: Parametrized Stokes equations, Reduced basis methods, Domain decomposition, Geometrical parametrization, Cardiovascular networks

\section{Introduction}

During the last decades a growing importance was given to cardiovascular fluid-dynamics as a key factor in describing some pathologies affecting the cardiovascular system $[2 ; 10]$.

Being able to perform simulations almost in real time and in many query context with a reasonable level of accuracy may increase the importance of cardiovascular simulations in daily diagnosis or risk evaluation procedure. In this range of applications, a big challenge is to speed up the computational time with rapid and efficient strategies that allow to approximate numerically fluid flows in complex and realistic configurations where topology features are recurrent and similar.

The reduced basis method is crucial to find the solution of parametrized problems as projection of previously precomputed solutions for certain instances of the parameters [25].

Thanks to the reduced basis method [20;21;34], reduced order strategies have been developed dealing with parametrized complex geometries and in order to take advantage of repetitive geometries occurring in the computational domain.

The reduced basis method applied to incompressible viscous flows in parametrized domain has been developed for Stokes equations in $[31 ; 32 ; 36]$, and more recently in [22], and for Navier-Stokes equations in $[7 ; 8 ; 9 ; 13 ; 23 ; 24 ; 28$; $35 ; 37$. Domain decomposition techniques are important to enable the use of parallel architectures in order to speed up the computational time, compared to a global approach, and also to face geometric complexity by dealing with independent smaller tasks on each subdomain, [30]. The treatment of the domain decomposed into several blocks and the reduced basis approach applied locally in each block has already been investigated for Stokes problem 
in $[16 ; 17]$, where the so-called reduced basis element method has been presented, and in [6] dealing with Maxwell's equations. A recent application of RBEM with new extensions as a static condensation method in heat transfer and solid mechanics problems is proposed in [12].

In this paper, some extensions of the reduced basis method are combined with decomposed domains to solve incompressible fluid flows problems modeled by steady Stokes equations. In particular our goal is to guarantee the continuity of velocity and stresses at the interfaces by proper coupling and gluing conditions, by the help of a coarse finite element solution. This aspect was not previously addressed with RBEM, where the recovery of the continuity of the velocity at the interface of the blocks is achieved by Lagrange multipliers, but normal stresses are not guaranteed to match continuously.

As for RBEM, the construction of the map from the reference subdomain to each reference block of the computational domain is carried out, as in [17], by the generalized transfinite map [18; 19]. The empirical interpolation procedure proposed in [3] has been applied to the geometrical non-affine transformation terms to recover affine properties for the decomposed operator.

In this work we start revisiting previous ideas about the reduced basis element method by considering the computational domain as an arbitrary union of non overlapping subdomains (blocks) which can be obtained as deformations of reference domains (reference blocks) [17]. Then, we present the reduced basis hybrid method by maintaining an offline and online computational splitting of the problem. The adjective hybrid is used here to underline that with respect to classical reduced basis methods (RB and RBEM) in this proposed approach we are using a coarse finite element solution as online correction to ensure continuity and consistency of the normal stress.

As in the reduced basis element method, the velocity continuity across block interfaces is guaranteed through the introduction of Lagrangian multipliers [16]. The original concept behind the reduced basis hybrid method is that the global solution, found by solving the Stokes problem, ensures not only the velocity continuity but also the continuity of normal stresses across block interfaces. Indeed, the final solution is a projection of local reduced basis with zero normal stress along the interfaces and a finite element solution computed in the whole computational domain with a very coarse grid, in order to guarantee the normal stress continuity at the interfaces. The coarse solution is computed by using an automatic assembling blocks algorithm, which is inexpensive and fast due to the small structures of the coarse meshes in 
the global network. This work is motivated by the fact that in several application (microfluidics and cardiovascular problems) also the pressure is a quantity of interest.

We provide here the outline of the paper. In Section 2 we recall the state equations represented by incompressible fluid flows modeled with steady Stokes equations, in Section 3 we introduce the parametrized formulation dealing with a multiple subdomains case. In Section 4 we recall the reduced basis formulation for a single subdomain and in Section 5 we extend the methodology with a reduced basis hybrid formulation of the problem (by combining reduced basis method and coarse finite element solution) for a multi-domain case. In Section 6 the transfinite map setting is introduced and finally in Section 7 we present some numerical results based on a series of multiply stenosed domains, an "heterogeneous" network configuration in a 2D setting, followed by some consideration on computational costs in Section 8. Then, an analysis of the computational complexity in the case of a geometry composed by an increasing number of blocks is considered in Section 9. Section 10 contains a test case dealing with a 3D parametrized stenosed configuration. Some considerations and conclusions follow in Section 11.

\section{Problem definition}

We consider the following steady Stokes problem in a domain $\Omega \subset \mathbb{R}^{2}$ with mixed boundary conditions on $\Gamma=\Gamma_{\text {in }} \cup \Gamma_{\text {out }} \cup \Gamma_{w}$ :

$$
\begin{cases}-\nu \Delta \mathbf{u}+\nabla p=\mathbf{f} & \text { in } \Omega, \\ \nabla \cdot \mathbf{u}=0 & \text { in } \Omega, \\ \mathbf{u}=0 & \text { on } \Gamma_{w} \\ \nu \frac{\partial \mathbf{u}}{\partial \mathbf{n}}-p \mathbf{n}=\overline{\boldsymbol{\sigma}}_{n}^{i n} & \text { on } \Gamma_{i n} \\ \nu \frac{\partial \mathbf{u}}{\partial \mathbf{n}}-p \mathbf{n}=\overline{\boldsymbol{\sigma}}_{n}^{\text {out }} & \text { on } \Gamma_{\text {out }}\end{cases}
$$

for a fluid of constant density; $\mathbf{u}$ is the fluid velocity, $p$ the pressure, $\mathbf{f}$ a force field (e.g. gravity), $\nu$ a kinematic viscosity and $\mathbf{n}$ the normal unit vector to the domain boundary; $\Gamma_{\text {in }}$ and $\Gamma_{\text {out }}$ represent the inflow and outflow, respectively, while $\Gamma_{w}$ is a boundary-wall. Here $\overline{\boldsymbol{\sigma}}_{n}^{\text {in }}$ and $\overline{\boldsymbol{\sigma}}_{n}^{\text {out }}$ represent imposed stresses on inflow and outflow, respectively.

On $\Omega$ we introduce the velocity space and the pressure space, respectively, 
as:

$$
Y=\left\{\mathbf{v} \in\left(H^{1}(\Omega)\right)^{2}:\left.\mathbf{v}\right|_{\Gamma_{w}}=\mathbf{0}\right\}, \quad M=L^{2}(\Omega) .
$$

Problem (1) in weak formulation reads: find $\mathbf{u} \in Y, p \in M$ :

$$
\begin{cases}a(\mathbf{u}, \mathbf{v})+b(\mathbf{v}, p)=F(\mathbf{v}) & \forall \mathbf{v} \in Y \\ b(\mathbf{u}, q)=0 & \forall q \in M\end{cases}
$$

where

$$
\begin{gathered}
a(\mathbf{v}, \mathbf{w})=\nu \int_{\Omega} \nabla \mathbf{v}: \nabla \mathbf{w} d \Omega=\nu \sum_{i, j=1}^{2} \int_{\Omega} \frac{\partial v_{i}}{\partial x_{j}} \frac{\partial w_{i}}{\partial x_{j}} d \Omega \\
b(\mathbf{v}, q)=-\int_{\Omega} q(\nabla \cdot \mathbf{v}) d \Omega=-\sum_{i=1}^{2} \int_{\Omega} q \frac{\partial v_{i}}{\partial x_{i}} d \Omega \\
F(\mathbf{v})=\int_{\Omega} \mathbf{f} \cdot \mathbf{v} d \Omega+\int_{\Gamma_{\text {in }}} \overline{\boldsymbol{\sigma}}_{n}^{\text {in }} \cdot \mathbf{v} d \Gamma+\int_{\Gamma_{\text {out }}} \overline{\boldsymbol{\sigma}}_{n}^{\text {out }} \cdot \mathbf{v} d \Gamma .
\end{gathered}
$$

The continuity of the bilinear forms $a(\cdot, \cdot)$ and $b(\cdot, \cdot)$, the coercivity condition on $a(\cdot, \cdot)$

$$
a(\mathbf{w}, \mathbf{w}) \geq \alpha\|\mathbf{w}\|_{H^{1}(\Omega)}^{2}, \quad \forall \mathbf{w} \in Y, \quad \alpha>0,
$$

and the inf-sup condition on $b(\cdot, \cdot)$

$$
\beta=\inf _{q \in M} \sup _{\mathbf{v} \in Y} \frac{b(\mathbf{v}, q)}{\|q\|_{L^{2}(\Omega)}\|\mathbf{v}\|_{H^{1}(\Omega)}}>0,
$$

allows to have the well posedness of problem (2) and ensure, thanks to the Brezzi theorem, the existence and uniqueness of the solution, see $[29 ; 26]$.

\section{The parametrized Stokes problem in local subdomain}

In this section we recall the parametrized Stokes problem by focusing on geometrical maps and the treatment of non-affine parametrization as already available in literature. In particular we introduce local Stokes problems in every subdomain of $\Omega$, this "decomposition" representing a crucial ingredient of the RBHM.

We assume that the domain $\Omega$ can be partitioned into a non-overlapping union of $R$ subdomains $\Omega_{r}$ and that each $\Omega_{r}$ is a deformation of a reference domain $\hat{\Omega}_{k(r)}$ through a regular enough, non-affine, map $T_{\boldsymbol{\mu}_{r}}^{k(r)}: \hat{\Omega}_{k(r)} \rightarrow \Omega_{r}$ 
so that:

$$
\begin{aligned}
\forall \hat{x} \in \hat{\Omega}_{k(r)}, x & =T_{\boldsymbol{\mu}_{r}}^{k(r)}\left(\boldsymbol{\mu}_{r}, \hat{x}\right), x \in \Omega_{r} \subset \Omega . \\
\bar{\Omega} & =\bigcup_{r=1}^{R} \bar{\Omega}_{r}=\bigcup_{r=1}^{R} \overline{T_{\boldsymbol{\mu}_{r}}^{k(r)}\left(\hat{\Omega}_{k(r)}\right)} .
\end{aligned}
$$

See Figure 1 for an example. A possible choice of these maps will be introduced in Section 6. The number of reference domain is $K \leq R$ (otherwise said, the map $k: \mathbb{N} \rightarrow \mathbb{N}, r \rightarrow k(r)$ is not necessarily injective). The same reference domain can serve the purpose for different subdomains, thanks to different choices of the parameter $\boldsymbol{\mu}_{r} \in \mathcal{D} \subset \mathbb{R}^{P}(P \geq 1)$, so that we can characterize different deformations of the same reference domain. In this sense we define a parametric map for each reference domain.

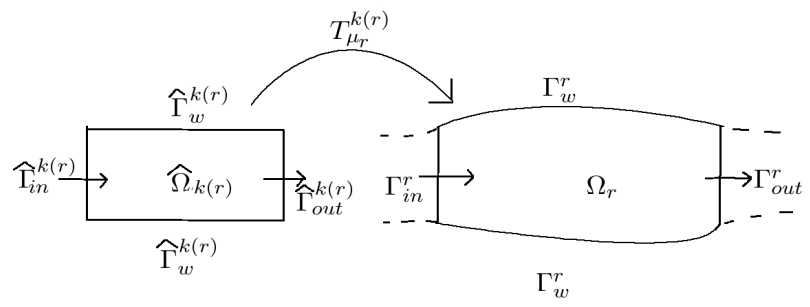

Figure 1: Scheme for a geometrical transformation from a reference domain.

For instance, on the example shown in Figure 2, we need only two reference domains, $\hat{\Omega}_{1}$ and $\hat{\Omega}_{2}$ and therefore only two parametric maps, $T_{\boldsymbol{\mu}_{r}}^{1}$ and $T_{\boldsymbol{\mu}_{r}}^{2}$. Then for any $\hat{x} \in \hat{\Omega}_{1}$ its image can define the four deformed bifurcations in $\Omega$, which are $\Omega_{4}, \Omega_{5}, \Omega_{6}$ and $\Omega_{7}$, through different choices of the parameter, respectively, $\boldsymbol{\mu}_{4}, \boldsymbol{\mu}_{5}, \boldsymbol{\mu}_{6}$ and $\boldsymbol{\mu}_{7}$. Any $\mathbf{x} \in \Omega_{4}$ is given by $T_{\boldsymbol{\mu}_{4}}^{1}(\hat{\mathbf{x}}):=T^{1}\left(\hat{\mathbf{x}}, \boldsymbol{\mu}_{4}\right), \forall \hat{\mathbf{x}} \in \hat{\Omega}_{1}$, while through $T_{\boldsymbol{\mu}_{r}}^{2}(\hat{\mathbf{x}})$ we can map the straight pipe reference domain $\hat{\Omega}_{2}$ in the deformed pipes $\Omega_{1}, \Omega_{2}, \Omega_{3}$ and $\Omega_{8}$, for suitable choices of the parameter $\boldsymbol{\mu}_{r}$, i.e. $\boldsymbol{\mu}_{1}, \boldsymbol{\mu}_{2}, \boldsymbol{\mu}_{3}, \boldsymbol{\mu}_{8}$ respectively.

For every $\Omega_{r}$ we denote by $\Gamma_{i n}^{r}$ its inflow boundary and by $\Gamma_{\text {out }}^{r}$ its outflow boundary, see Figure 1. We call $\Omega_{r}$ an "inflow" element if $\Gamma_{i n}^{r} \subset \Gamma_{\text {in }}$, "outflow" element if $\Gamma_{\text {out }}^{r} \subset \Gamma_{\text {out }}$, and "central" element when $\Gamma^{r} \cap \Gamma=\Gamma_{w}^{r}$, here $\Gamma_{\text {in }}, \Gamma_{\text {out }}$ and $\Gamma$ denote the global inflow, outflow and the boundaries walls of $\Omega$. In the example illustrated in Figure $2, \Omega_{1}$ is an "inflow" element, $\Omega_{r}$, with $r=2,3,4,6$ are "central" elements and $\Omega_{8}, \Omega_{7}, \Omega_{5}$ are "outflow" elements. Note that for more complex configurations involving central subdomains with 


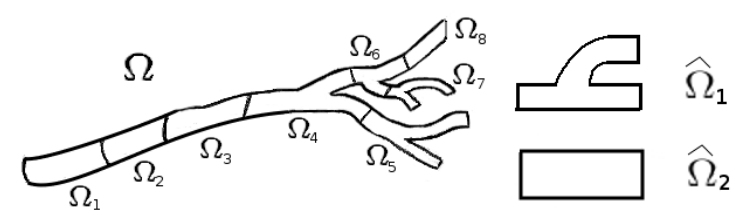

Figure 2: A domain composed by $\mathrm{R}=8$ blocks that can be obtained as deformation of $\mathrm{K}=2$ reference domains.

either inflow or outflow boundaries, more elements should be considered.

We denote with $\boldsymbol{\sigma}^{r}$ and $\hat{\boldsymbol{\sigma}}^{k(r)}$ the Cauchy stress tensors on $\Omega_{r}$ and $\hat{\Omega}_{k(r)}$, respectively, and with $\boldsymbol{\sigma}_{n}^{r}=\boldsymbol{\sigma}^{r} \mathbf{n}^{r}$ and $\hat{\boldsymbol{\sigma}}_{n}^{k(r)}=\hat{\boldsymbol{\sigma}}^{k(r)} \hat{\mathbf{n}}^{k(r)}$ the imposed stresses on inflow and outflow boundaries, where $\mathbf{n}^{r}$ and $\hat{\mathbf{n}}^{k(r)}$ are the unit outward normal vectors on $\partial \Omega_{r}$ and $\partial \hat{\Omega}_{k(r)}$, respectively.

We introduce now the formulation of the Stokes problem in every generic deformation of $\hat{\Omega}_{k(r)}$. As shown in $[15 ; 17]$, the parametric maps $T_{\boldsymbol{\mu}_{r}}^{k(r)}$ and their Jacobians $\boldsymbol{J}_{k(r)}$ allow the definition of the bilinear and linear forms on a deformed domain, $\Omega_{r}\left(=T_{\boldsymbol{\mu}_{r}}^{k(r)}\left(\hat{\Omega}_{k(r)}\right)\right)$, through the evaluation of the corresponding forms in the reference domain $\hat{\Omega}_{k(r)}$, that is:

$$
\begin{gathered}
a^{k(r)}\left(\hat{\mathbf{v}}, \hat{\mathbf{w}}, \boldsymbol{\mu}_{r}\right)=\nu \int_{\hat{\Omega}_{k(r)}} \boldsymbol{J}_{k(r)}^{-T} \nabla \hat{\mathbf{v}}: \boldsymbol{J}_{k(r)}^{-T} \nabla \hat{\mathbf{w}}\left|\boldsymbol{J}_{k(r)}\right| d \hat{\Omega}_{k(r)}, \\
b^{k(r)}\left(\hat{\mathbf{v}}, \hat{q}, \boldsymbol{\mu}_{r}\right)=-\int_{\hat{\Omega}_{k(r)}} \hat{q} \nabla \cdot\left(\boldsymbol{J}_{k(r)}^{-1} \hat{\mathbf{v}}\right)\left|\boldsymbol{J}_{k(r)}\right| d \hat{\Omega}_{k(r)}, \\
F^{k(r)}\left(\hat{\mathbf{w}}, \boldsymbol{\mu}_{r}, \hat{\boldsymbol{\sigma}}_{n}^{k(r)}\right)=\int_{\hat{\Omega}_{k(r)}} \hat{\mathbf{f}} \cdot \hat{\mathbf{v}}\left|\boldsymbol{J}_{k(r)}\right| d \hat{\Omega}_{k(r)}+\int_{\hat{\Gamma}_{i n}^{k(r)} \cup \hat{\Gamma}_{\text {out }}^{k(r)}} \hat{\boldsymbol{\sigma}}_{n}^{k(r)} \cdot \hat{\mathbf{v}}\left|\boldsymbol{J}_{k(r)}\right| d \hat{\Gamma}_{\hat{\Omega}_{k(r)}} .
\end{gathered}
$$

We impose no-slip boundary condition on $\hat{\Gamma}_{w}^{k(r)}$ and Neumann boundary conditions on $\hat{\Gamma}_{i n}^{k(r)}$ and $\hat{\Gamma}_{\text {out }}^{k(r)}$ with:

- $\left.\hat{\boldsymbol{\sigma}}_{n}^{k(r)}\right|_{\hat{\Gamma}_{i n}^{k(r)}}=\overline{\boldsymbol{\sigma}}_{n}^{i n}$ and $\left.\hat{\boldsymbol{\sigma}}_{n}^{k(r)}\right|_{\hat{\Gamma}_{\text {out }}^{k(r)}}=\mathbf{0}$, if $\Omega_{r}=T_{\boldsymbol{\mu}_{r}}^{k(r)}\left(\hat{\Omega}_{k(r)}\right)$ is an "inflow" element;

- $\left.\hat{\boldsymbol{\sigma}}_{n}^{k(r)}\right|_{\hat{\Gamma}_{\text {in }}^{k(r)} \cup \hat{\Gamma}_{\text {out }}^{k(r)}}=\mathbf{0}$, if $\Omega_{r}$ is a "central" element;

- $\left.\hat{\boldsymbol{\sigma}}_{n}^{k(r)}\right|_{\hat{\Gamma}_{i n}^{k(r)}}=\mathbf{0}$ and $\left.\hat{\boldsymbol{\sigma}}_{n}^{k(r)}\right|_{\hat{\Gamma}_{\text {out }}^{k(r)}}=\overline{\boldsymbol{\sigma}}_{n}^{\text {out }}$, if $\Omega_{r}$ is an "outflow" element. 
In case of homogeneous boundary data and null force term, a local Stokes problem might feature the null solution.

The Piola transformation can be used to transform the velocity field in order to maintain the orientation of the velocity with respect to the inflow and outflow boundaries, $[4 ; 8]$. Alternatively, we can get rid of this transformation by collecting the contributions from the transposed inverse Jacobians and the Jacobian determinant in the tensors $\boldsymbol{\nu}^{k(r)}$ and $\boldsymbol{\chi}^{k(r)}$, for viscous and pressure terms, respectively, and use the elements of this tensor as the parameter dependent functions. This mentioned procedure is adopted in this paper and it is convenient as long as the outward normal vectors on the inflow and outflow boundaries are preserved after the geometrical deformations of the domain. The transformation tensors for the bilinear forms $a^{k(r)}\left(\cdot, \cdot, \boldsymbol{\mu}_{r}\right)$ and $b^{k(r)}\left(\cdot, \cdot, \boldsymbol{\mu}_{r}\right)$ are:

$$
\boldsymbol{\nu}^{k(r)}\left(\hat{\mathbf{x}}, \boldsymbol{\mu}_{r}\right)=\boldsymbol{J}_{k(r)}^{-1} \boldsymbol{J}_{k(r)}^{-T}\left|\boldsymbol{J}_{k(r)}\right|
$$

and

$$
\chi^{k(r)}\left(\hat{\mathbf{x}}, \boldsymbol{\mu}_{r}\right)=\boldsymbol{J}_{k(r)}^{-1}\left|\boldsymbol{J}_{k(r)}\right| .
$$

We can express (6) and (7) as

$$
\begin{gathered}
a^{k(r)}\left(\hat{\mathbf{v}}, \hat{\mathbf{w}}, \boldsymbol{\mu}_{r}\right)=\nu \int_{\hat{\Omega}_{k(r)}} \boldsymbol{\nu}^{k(r)}\left(\hat{\mathbf{x}}, \boldsymbol{\mu}_{r}\right) \nabla \hat{\mathbf{v}}: \nabla \hat{\mathbf{w}} d \hat{\Omega}_{k(r)}, \\
b^{k(r)}\left(\hat{\mathbf{v}}, \hat{q}, \boldsymbol{\mu}_{r}\right)=\int_{\hat{\Omega}_{k(r)}} \chi^{k(r)}\left(\hat{\mathbf{x}}, \boldsymbol{\mu}_{r}\right) \hat{q} \nabla \cdot \hat{\mathbf{v}} d \hat{\Omega}_{k(r)} .
\end{gathered}
$$

Since the tensors $\boldsymbol{\nu}^{k(r)}(\hat{\mathbf{x}}, \boldsymbol{\mu}), \boldsymbol{\chi}^{k(r)}(\hat{\mathbf{x}}, \boldsymbol{\mu})$ and the determinant $\left|\boldsymbol{J}_{k(r)}(\hat{\mathbf{x}}, \boldsymbol{\mu})\right|$ are non-affine for $k(r)=1, \cdots, K$, we apply the empirical interpolation procedure [3] to decompose each component of these tensors in parameter dependent contribution and other parts depending only on spatial coordinates [32]. The idea is to approximate the parameter dependent components $\left[\boldsymbol{\nu}^{k(r)}(\hat{\mathbf{x}}, \boldsymbol{\mu})\right]_{i j},\left[\boldsymbol{\chi}^{k(r)}(\hat{\mathbf{x}}, \boldsymbol{\mu})\right]_{i j}$ as well as the determinant $\left|\boldsymbol{J}_{k(r)}(\hat{\mathbf{x}}, \boldsymbol{\mu})\right|$, as linear combination of a few "basis" functions, $\tilde{\boldsymbol{\nu}}_{i j}^{k(r) m}(\hat{\mathbf{x}})=\left[\boldsymbol{\nu}^{k(r)}\left(\hat{\mathbf{x}}, \boldsymbol{\mu}_{m}\right)\right]_{i j}, \tilde{\boldsymbol{\chi}}_{i j}^{k(r) n}(\hat{\mathbf{x}})=$ $\left[\boldsymbol{\chi}^{k(r)}\left(\hat{\mathbf{x}}, \boldsymbol{\mu}_{n}\right)\right]_{i j}$ and $\tilde{J}_{k(r) s}(\hat{\mathbf{x}})=\left|\boldsymbol{J}_{k(r)}\left(\hat{\mathbf{x}}, \boldsymbol{\mu}_{s}\right)\right|$, where $\boldsymbol{\mu}_{m}, \boldsymbol{\mu}_{n}$ and $\boldsymbol{\mu}_{s}$ are properly selected within a predefined set of sampling parameters, as introduced in $[3]$.

We can decouple parameter dependent coefficients from a parameter independent part thanks to the following expansions (no summation on repeated 
indices here):

$$
\begin{aligned}
{\left[\boldsymbol{\nu}^{k(r)}(\hat{\mathbf{x}}, \boldsymbol{\mu})\right]_{i j} } & =\sum_{m=1}^{M_{i j}^{a k(r)}} \Theta_{i j}^{k(r) m}(\boldsymbol{\mu}) \tilde{\boldsymbol{\nu}}_{i j}^{k(r) m}(\hat{\mathbf{x}})+\boldsymbol{\epsilon}_{i j}^{a k(r)}(\hat{\mathbf{x}}, \boldsymbol{\mu}), \\
{\left[\boldsymbol{\chi}^{k(r)}(\hat{\mathbf{x}}, \boldsymbol{\mu})\right]_{i j} } & =\sum_{n=1}^{M_{i j}^{b k(r)}} \Phi_{i j}^{k(r) n}(\boldsymbol{\mu}) \tilde{\boldsymbol{\chi}}_{i j}^{k(r) n}(\hat{\mathbf{x}})+\boldsymbol{\epsilon}_{i j}^{b k(r)}(\hat{\mathbf{x}}, \boldsymbol{\mu}), \\
\left|\boldsymbol{J}_{k}(\hat{\mathbf{x}}, \boldsymbol{\mu})\right| & =\sum_{s=1}^{M^{s k(r)}} \Psi^{k(r) s}(\boldsymbol{\mu}) \tilde{J}_{k(r) s}(\hat{\mathbf{x}})+\boldsymbol{\epsilon}^{s k(r)}(\hat{\mathbf{x}}, \boldsymbol{\mu}) .
\end{aligned}
$$

In the previous expressions $\Theta_{i j}^{k(r) m}, \Phi_{i j}^{k(r) n}, \Psi^{k(r) s}: \mathcal{D} \rightarrow \mathbb{R}$ are weighing quantities depending on the parameters; $\tilde{\boldsymbol{\nu}}_{i j}^{k(r) m}, \tilde{\boldsymbol{\chi}}_{i j}^{k(r) n}, \tilde{J}_{k(r) s}$ are interpolation functions used as basis, $M$ refers to the number of interpolation functions we use for each form and it is related with the maximum interpolation error $\epsilon_{\text {tol }}^{E I M}$, such that:

$$
\begin{gathered}
\left\|\boldsymbol{\epsilon}_{i j}^{a k(r)}(\cdot, \cdot)\right\|_{\infty} \leq \epsilon_{t o l}^{E I M}, \quad i, j=1,2, \\
\left\|\boldsymbol{\epsilon}_{i j}^{b k(r)}(\cdot, \cdot)\right\|_{\infty} \leq \epsilon_{t o l}^{E I M}, \quad i, j=1,2, \\
\left\|\boldsymbol{\epsilon}^{s k(r)}(\cdot, \cdot)\right\|_{\infty} \leq \epsilon_{t o l}^{E I M} .
\end{gathered}
$$

By applying this affine decomposition to the terms (8), (11) and (12) we define the following linear and bilinear forms as their respective approximations:

$$
\begin{gathered}
\mathcal{F}^{k(r)}\left(\hat{\mathbf{v}}, \boldsymbol{\mu}, \hat{\boldsymbol{\sigma}}_{n}^{k(r)}\right)=\sum_{s=1}^{M^{s k(r)}} \Psi^{k(r) s}(\boldsymbol{\mu}) \mathcal{F}^{k(r) s}\left(\hat{\mathbf{v}}, \hat{\boldsymbol{\sigma}}_{n}^{k(r)}\right), \\
\mathcal{A}^{k(r)}(\hat{\mathbf{v}}, \hat{\mathbf{w}}, \boldsymbol{\mu})=\nu \sum_{i=1}^{2} \sum_{j=1}^{2} \sum_{m=1}^{M_{i j}^{a k(r)}} \Theta_{i j}^{k(r) m}(\boldsymbol{\mu}) \mathcal{A}_{i j}^{k(r) m}(\hat{\mathbf{v}}, \hat{\mathbf{w}}), \\
\mathcal{B}^{k(r)}(\hat{\mathbf{v}}, \hat{q}, \boldsymbol{\mu})=\sum_{i=1}^{2} \sum_{j=1}^{2} \sum_{n=1}^{M_{i j}^{b k(r)}} \Phi_{i j}^{k(r) n}(\boldsymbol{\mu}) \mathcal{B}_{i j}^{k(r) n}(\hat{\mathbf{v}}, \hat{q}),
\end{gathered}
$$


where

$$
\begin{aligned}
\mathcal{F}^{k(r) m}\left(\hat{\mathbf{v}}, \hat{\boldsymbol{\sigma}}_{n}^{k(r)}\right) & =\int_{\hat{\Omega}_{k(r)}} \tilde{J}_{k(r) m}(\hat{\mathbf{x}}) \hat{\mathbf{f}} \cdot \hat{\mathbf{v}} d \hat{\Omega}_{k(r)}+\int_{\hat{\Gamma}_{i n}^{k(r)} \cup \hat{\Gamma}_{o u t}^{k(r)}} \tilde{J}_{k m}(\hat{\mathbf{x}}) \hat{\boldsymbol{\sigma}}_{n}^{k(r)} \hat{\mathbf{v}} d \hat{\Gamma}_{\hat{\Omega}_{k(r)}}, \\
\mathcal{A}_{i j}^{k(r) m}(\hat{\mathbf{v}}, \hat{\mathbf{w}}) & =\int_{\hat{\Omega}_{k(r)}} \tilde{\boldsymbol{\nu}}_{i j}^{k(r) m}(\hat{\mathbf{x}}) \frac{\partial \hat{\mathbf{v}}}{\partial \hat{x}_{i}} \frac{\partial \hat{\mathbf{w}}_{i}}{\partial \hat{x}_{j}} d \hat{\Omega}_{k(r)}, \\
\mathcal{B}_{i j}^{k(r) m}(\hat{\mathbf{v}}, \hat{q}) & =-\int_{\hat{\Omega}_{k(r)}} \tilde{\boldsymbol{\chi}}_{i j}^{k(r) m}(\hat{\mathbf{x}}) \hat{q} \frac{\partial \hat{v}_{i}}{\partial \hat{x}_{j}} d \hat{\Omega}_{k(r)} .
\end{aligned}
$$

This affine decomposition will be useful in the reduced basis method to split all the heavy computation involving high resolution (concerning discretization) in an offline stage and, then during an online stage, to solve efficiently the problem for each new choice of the parameters and for each subdomain that we want to consider in the network configuration.

For a new $\boldsymbol{\mu}_{r}$ and for the proper reference domain $\hat{\Omega}_{k(r)}$ the Stokes problem can be rewritten as: find $\left(\hat{\mathbf{u}}\left(\boldsymbol{\mu}_{r}\right), \hat{p}\left(\boldsymbol{\mu}_{r}\right)\right) \in Y^{k(r)} \times M^{k(r)}$ such that

$$
\begin{cases}\mathcal{A}^{k(r)}\left(\hat{\mathbf{u}}\left(\boldsymbol{\mu}_{r}\right), \hat{\mathbf{w}}, \boldsymbol{\mu}_{r}\right)+\mathcal{B}^{k(r)}\left(\hat{\mathbf{w}}, \hat{p}\left(\boldsymbol{\mu}_{r}\right), \boldsymbol{\mu}_{r}\right)=\mathcal{F}^{k(r)}\left(\hat{\mathbf{w}}, \boldsymbol{\mu}_{r}, \hat{\boldsymbol{\sigma}}_{n}^{k(r)}\right) & \forall \hat{\mathbf{w}} \in Y^{k(r)}, \\ \mathcal{B}^{k(r)}\left(\hat{\mathbf{u}}\left(\boldsymbol{\mu}_{r}\right), \hat{q}, \boldsymbol{\mu}_{r}\right)=0 & \forall \hat{q} \in M^{k(r)},\end{cases}
$$

where

$$
\begin{aligned}
& Y^{k(r)}=\left\{\hat{\mathbf{v}} \in\left(H^{1}\left(\hat{\Omega}_{k(r)}\right)\right)^{2}:\left.\hat{\mathbf{v}}\right|_{\hat{\Gamma}_{w}^{k(r)}}=0\right\}, \quad \hat{\Gamma}_{w}^{k(r)}=T_{\boldsymbol{\mu}_{r}}^{k(r)^{-1}}\left(\Gamma_{w} \cap \partial \Omega_{r}\right), \\
& M^{k(r)}=L^{2}\left(\hat{\Omega}_{k(r)}\right) .
\end{aligned}
$$

The solution of this problem satisfies the inf-sup condition [29], expressed by the following inequality:

$$
\begin{aligned}
\exists \beta_{0}^{k(r)}>0: \beta^{k(r)}\left(\boldsymbol{\mu}_{r}\right):=\inf _{\hat{q} \in M^{k(r)}} \sup _{\hat{w} \in Y^{k(r)}} \frac{\mathcal{B}^{k(r)}\left(\hat{\mathbf{w}}, \hat{q}, \boldsymbol{\mu}_{r}\right)}{\|\hat{\mathbf{w}}\|_{Y^{k(r)}}\|\hat{q}\|_{M^{k(r)}}} \geq \beta_{0}^{k(r)}, \\
\forall \boldsymbol{\mu}_{r} \in \mathcal{D}, \forall k(r)=1, \cdots, K .
\end{aligned}
$$

In particular, starting from some reference domains, problem (16) represents a (local) well-posed Stokes problem in each deformed block of the computational domain, which accounts for imposing proper Neumann boundary conditions that are dictated by the relative "position" of the deformed subdomain (inflow, outflow or central).

Once we have the local Stokes formulations, we can find the Stokes solution in the global domain $\Omega$ through suitable assumptions that will be introduced in the next sections together with the reduced basis formulation. 


\section{The reduced basis formulation}

We start recalling the classical reduced basis formulation for a single domain case and then we extend it to a multi-domain case. Since we are considering only one reference domain $\hat{\Omega}$, we can omit the $k$ index and, from now we omit the "hat" to further simplify the notations, however we warn the reader that we are always referring to the reference subdomain. For given Neumann boundary condition, we look for a reduced basis formulation of problem (16). With this aim we build a set of parameter samples $S_{N}^{\boldsymbol{\mu}}=\left\{\boldsymbol{\mu}^{1}, \cdots, \boldsymbol{\mu}^{N}\right\}$ and correspondingly a set of pairs $\left(\mathbf{u}_{h}\left(\boldsymbol{\mu}^{i}\right), p_{h}\left(\boldsymbol{\mu}^{i}\right)\right)$ which are approximate solutions of the Stokes problem using Galerkin Finite Element method on an accurate fine mesh $\mathcal{T}_{h}$, where as customary $h$ indicates the maximum edge size of $\mathcal{T}_{h}$. The choice of the parameter set $S_{N}^{\mu}$ has been done using a greedy algorithm, like those proposed in $[31 ; 32]$.

Following $[31 ; 32 ; 36]$, an approximation of problem (16) is computed as a Galerkin projection onto the following low dimensional subspaces $Z_{N}$ and $M_{N}$ for velocity and pressure, respectively:

$$
\begin{gathered}
Z_{N}=\operatorname{span}\left\{\mathbf{u}_{h}\left(\boldsymbol{\mu}^{i}\right), i=1, \ldots, N\right\}, \\
M_{N}=\operatorname{span}\left\{p_{h}\left(\boldsymbol{\mu}^{i}\right), i=1, \ldots, N\right\} .
\end{gathered}
$$

In order to guarantee the approximation stability of the reduced basis method for Stokes problem, we fulfill the inf-sup condition (17) by enriching the velocity basis as follows. For every pressure solution $p_{h}\left(\boldsymbol{\mu}^{i}\right)$ spanning $M_{N}$, we define:

$$
\mathbf{v}_{h}\left(\boldsymbol{\mu}^{i}\right)=\arg \sup _{\mathbf{w} \in Z} \frac{\mathcal{B}\left(\mathbf{w}, p_{h}\left(\boldsymbol{\mu}^{i}\right), \boldsymbol{\mu}^{i}\right)}{\|\mathbf{w}\|_{Z}},
$$

and then

$$
X_{N}=\operatorname{span}\left\{\mathbf{v}_{h}\left(\boldsymbol{\mu}^{i}\right), i=1, \ldots, N\right\} .
$$

Finally, the enriched velocity space is defined by:

$$
Y_{N}=Z_{N} \oplus X_{N}
$$

By setting

$$
\beta_{N}(\boldsymbol{\mu}):=\inf _{q \in M_{N}} \sup _{\mathbf{w} \in Y_{N}} \frac{\mathcal{B}(\mathbf{w}, q, \boldsymbol{\mu})}{\|\mathbf{w}\|_{Y}\|q\|_{M}} \geq \beta_{0}>0 \quad \forall \boldsymbol{\mu} \in \mathcal{D}
$$


as shown in [36] and more recently in [33], the following condition, binding (17) and (22), is fulfilled:

$$
\beta_{N}(\boldsymbol{\mu}) \geq \beta(\boldsymbol{\mu}) \geq \beta_{0}>0 \quad \forall \boldsymbol{\mu} \in \mathcal{D}
$$

where $\beta(\boldsymbol{\mu})$ is the inf-sup constant (17) related to the Galerkin Finite Element method.

The reduced basis approximation of problem (16) reads: find $\left(\mathbf{u}_{N}(\boldsymbol{\mu}), p_{N}(\boldsymbol{\mu})\right) \in$ $\left(Y_{N} \times M_{N}\right)$ such that

$$
\begin{cases}\mathcal{A}\left(\mathbf{u}_{N}(\boldsymbol{\mu}), \mathbf{w}, \boldsymbol{\mu}\right)+\mathcal{B}\left(\mathbf{w}, p_{N}(\boldsymbol{\mu}), \boldsymbol{\mu}\right)=\mathcal{F}\left(\mathbf{w}, \boldsymbol{\mu}, \boldsymbol{\sigma}_{n}\right) & \forall \mathbf{w} \in Y_{N} \\ \mathcal{B}\left(\mathbf{u}_{N}(\boldsymbol{\mu}), q, \boldsymbol{\mu}\right)=0 & \forall q \in M_{N}\end{cases}
$$

Note that this represents the generic RB formulation of the Stokes problem (16), with $\boldsymbol{\sigma}_{n}$ representing the proper imposed stress on the inflow and on the outflow. By writing:

$$
\begin{gathered}
\mathbf{u}_{N}(\boldsymbol{\mu})=\sum_{i=1}^{N} u_{N i}(\boldsymbol{\mu}) \mathbf{u}_{h}\left(\boldsymbol{\mu}^{i}\right)+\sum_{i=N+1}^{2 N} u_{N i}(\boldsymbol{\mu}) \mathbf{v}_{h}\left(\boldsymbol{\mu}^{i}\right) \\
p_{N}(\boldsymbol{\mu})=\sum_{i=1}^{N} p_{N i}(\boldsymbol{\mu}) p_{h}\left(\boldsymbol{\mu}^{i}\right),
\end{gathered}
$$

we find that the coefficients $u_{N i}$ and $p_{N i}$ are obtained by solving the following linear system:

$$
\begin{cases}\sum_{i=1}^{2 N} A_{j i}^{\boldsymbol{\mu}} u_{N i}(\boldsymbol{\mu})+\sum_{i=1}^{N} B_{j i}^{\boldsymbol{\mu}} p_{N i}(\boldsymbol{\mu})=F_{j}^{\boldsymbol{\mu}} & 1 \leq j \leq 2 N \\ \sum_{i=1}^{2 N} B_{i j}^{\boldsymbol{\mu}} u_{N i}(\boldsymbol{\mu})=0 & 1 \leq j \leq N\end{cases}
$$

where

$$
\begin{gathered}
A_{i j}^{\boldsymbol{\mu}}=\mathcal{A}\left(\mathbf{w}_{i}, \mathbf{w}_{j}, \boldsymbol{\mu}\right), \quad B_{i l}^{\boldsymbol{\mu}}=\mathcal{B}\left(\mathbf{w}_{i}, p_{l}, \boldsymbol{\mu}\right), \quad F_{j}^{\boldsymbol{\mu}}=\mathcal{F}\left(\mathbf{w}_{j}, \boldsymbol{\mu}, \boldsymbol{\sigma}_{n}\right), \\
1 \leq i, j \leq 2 N, \quad 1 \leq l \leq N, \quad \mathbf{w}_{i}, \mathbf{w}_{j} \in Y_{N}, \quad p_{k} \in M_{N} .
\end{gathered}
$$

Since the bilinear forms are now affinely parametrized (after the empirical interpolation treatment), in an offline expensive stage we can compute the parameter independent parts of the matrices $A^{\mu}, B^{\mu}$ and the vector $F^{\mu}$ (that include FE matrices, basis functions and pre and post multiplication 
procedures of the FE matrices for the basis functions computed). Then in an online stage, for each new parameter value the parametric coefficients of the system can be quickly evaluated. Finally, a small linear system can be solved efficiently during the online stage many times to find the coefficients $u_{N i}$ and $p_{N i}$ that will give the final reduced basis solution for each new value of $\boldsymbol{\mu}[33]$.

\section{The reduced basis hybrid method}

What recalled in Sections 3 and 4 is a framework common to both RB method and RBEM, in this Section we formulate the proposed variant of these methods: the reduced basis hybrid method (RBHM) for computational domains with rigid boundaries. Before providing a general description of the method and its computationally efficient realization, we illustrate the basic concept on a simplified case.

\subsection{Two domains with single internal interface}

We consider a domain $\Omega$ parametrized through $\boldsymbol{\mu}=\left(\boldsymbol{\mu}_{1}, \boldsymbol{\mu}_{2}\right)$ and its subdomain decomposition in $\Omega_{1}$, parametrized through $\boldsymbol{\mu}_{1}$, and $\Omega_{2}$ parametrized through $\boldsymbol{\mu}_{2} . \Gamma_{12}$ is the common interface.

We want to solve the following Stokes problem on $\Omega$ : find $(\mathbf{u}(\boldsymbol{\mu}), p(\boldsymbol{\mu}))$ such that

$$
\begin{cases}\mathcal{A}(\mathbf{u}(\boldsymbol{\mu}), \mathbf{w}, \boldsymbol{\mu})+\mathcal{B}(\mathbf{w}, p(\boldsymbol{\mu}), \boldsymbol{\mu})=\mathcal{F}(\mathbf{w}, \boldsymbol{\mu}) & \forall \mathbf{w} \in Y, \\ \mathcal{B}(\mathbf{u}(\boldsymbol{\mu}), q, \boldsymbol{\mu})=0 & \forall q \in M,\end{cases}
$$

where the source term $\mathcal{F}(\mathbf{w}, \boldsymbol{\mu})$ accounts the normal stresses $\overline{\boldsymbol{\sigma}}_{n}^{\text {in }}$ on $\Gamma_{\text {in }}$ and $\overline{\boldsymbol{\sigma}}_{n}^{\text {out }}$ on $\Gamma_{\text {out }}$, i.e. on the inflow and the outflow, respectively. Thus $\mathcal{F}(\mathbf{w}, \boldsymbol{\mu})=$ $\mathcal{F}\left(\mathbf{w}, \boldsymbol{\mu}, \overline{\boldsymbol{\sigma}}_{n}\right)$. We denote $(25)$ as $S t_{\Omega}(\mathbf{u}(\boldsymbol{\mu}), p(\boldsymbol{\mu}), \boldsymbol{\mu})=0$.

We consider now the restriction of $(25)$ to $\Omega_{1}$ and $\Omega_{2}$ by maintaining the boundary condition on $\Gamma_{\text {in }} \subset \partial \Omega_{1}$, and on $\Gamma_{\text {out }} \subset \partial \Omega_{2}$. Besides, we impose zero normal stress on the boundary $\Gamma_{12}$ for either problem. We denote the two corresponding independent Stokes problems with $S t_{\Omega_{1}}\left(\mathbf{u}^{1}\left(\boldsymbol{\mu}_{1}\right), p^{1}\left(\boldsymbol{\mu}_{1}\right), \boldsymbol{\mu}_{1}\right)=$ 0 and $S t_{\Omega_{2}}\left(\mathbf{u}^{2}\left(\boldsymbol{\mu}_{2}\right), p^{2}\left(\boldsymbol{\mu}_{2}\right), \boldsymbol{\mu}_{2}\right)=0$.

For either problem, according to a standard RB procedure, we select a set of representative sampling parameters, say $\boldsymbol{\mu}_{1}^{j}, j=1, \cdots, N_{1}$ and $\boldsymbol{\mu}_{2}^{j}, j=$ $1, \cdots, N_{2}$, respectively. Correspondingly, we compute (offline) the following set of basis functions on a fine accurate mesh $\mathcal{T}_{h}$ :

$$
\left\{\mathbf{u}_{h}^{1}\left(\boldsymbol{\mu}_{1}^{j}\right), \mathbf{v}_{h}^{1}\left(\boldsymbol{\mu}_{1}^{j}\right), p_{h}^{1}\left(\boldsymbol{\mu}_{1}^{j}\right), j=1, \cdots, N_{1}\right\} \text { on } \Omega_{1},
$$




$$
\left\{\mathbf{u}_{h}^{2}\left(\boldsymbol{\mu}_{2}^{j}\right), \mathbf{v}_{h}^{2}\left(\boldsymbol{\mu}_{2}^{j}\right), p_{h}^{2}\left(\boldsymbol{\mu}_{2}^{j}\right), j=1, \cdots, N_{2}\right\} \text { on } \Omega_{2},
$$

where $\mathbf{u}_{h}^{1}\left(\boldsymbol{\mu}_{1}^{j}\right), p_{h}^{1}\left(\boldsymbol{\mu}_{1}^{j}\right)$ is the FE solution of $S t_{\Omega_{1}}\left(\mathbf{u}_{h}^{1}\left(\boldsymbol{\mu}_{1}^{j}\right), p_{h}^{1}\left(\boldsymbol{\mu}_{1}^{j}\right), \boldsymbol{\mu}_{1}^{j}\right)=0$, $\mathbf{u}_{h}^{2}\left(\boldsymbol{\mu}_{2}^{j}\right), p_{h}^{2}\left(\boldsymbol{\mu}_{2}^{j}\right)$ the FE solution of $S t_{\Omega_{2}}\left(\mathbf{u}_{h}^{2}\left(\boldsymbol{\mu}_{2}^{j}\right), p_{h}^{2}\left(\boldsymbol{\mu}_{2}^{j}\right), \boldsymbol{\mu}_{2}^{j}\right)=0$, and $\mathbf{v}_{h}^{1}\left(\boldsymbol{\mu}_{1}^{j}\right)$, $\mathbf{v}_{h}^{2}\left(\boldsymbol{\mu}_{2}^{j}\right)$ the associated velocity supremizers. Note that the normal stresses vanish (by construction) on $\Gamma_{12}$ (whence they match continuously, that is their jump is zero) whereas the velocities are (in principle) discontinuous across $\Gamma_{12}$.

We move now online, that is we consider a specific given value of the parameter $\boldsymbol{\mu}$. Correspondingly, we compute an approximation of the whole problem (25), say $S t_{\Omega}(\mathbf{u}(\boldsymbol{\mu}), p(\boldsymbol{\mu}), \boldsymbol{\mu})=0$, on a (very) coarse grid $\mathcal{T}_{H}$. Let us indicate the corresponding FE coarse solution as $\mathbf{u}_{H}(\boldsymbol{\mu}), p_{H}(\boldsymbol{\mu})$, and by $\mathbf{v}_{H}(\boldsymbol{\mu})$ the corresponding velocity supremizer.

Clearly, this coarse solution fulfills all the desired continuities at the interface $\Gamma_{12}$ that is both $\mathbf{u}_{H}(\boldsymbol{\mu})$ and $\mathbf{v}_{H}(\boldsymbol{\mu})$, as well as their associated normal stresses, are continuous.

At this stage, our RBHM solution is sought (online) by proceeding as follows. We look for a suitable linear combination of the previously computed solutions, that is:

$$
\begin{gathered}
\mathbf{u}_{\left.N\right|_{\Omega_{1}}}(\boldsymbol{\mu})=\sum_{j=1}^{N_{1}}\left(\alpha_{j}^{1} \mathbf{u}_{h}^{1}\left(\boldsymbol{\mu}_{1}^{j}\right)+\beta_{j}^{1} \mathbf{v}_{h}^{1}\left(\boldsymbol{\mu}_{1}^{j}\right)\right)+\eta \mathbf{u}_{H}^{1}(\boldsymbol{\mu})+\delta \mathbf{v}_{H}^{1}(\boldsymbol{\mu}), \\
p_{\left.N\right|_{\Omega_{1}}}(\boldsymbol{\mu})=\sum_{j=1}^{N_{1}} \gamma_{j}^{1} p_{h}^{1}\left(\boldsymbol{\mu}_{1}^{j}\right)+\epsilon p_{H}^{1}(\boldsymbol{\mu}), \\
\mathbf{u}_{\left.N\right|_{\Omega_{2}}}(\boldsymbol{\mu})=\sum_{j=1}^{N_{2}}\left(\alpha_{j}^{2} \mathbf{u}_{h}^{2}\left(\boldsymbol{\mu}_{2}^{j}\right)+\beta_{j}^{2} \mathbf{v}_{h}^{2}\left(\boldsymbol{\mu}_{2}^{j}\right)\right)+\eta \mathbf{u}_{H}^{2}(\boldsymbol{\mu})+\delta \mathbf{v}_{H}^{2}(\boldsymbol{\mu}), \\
p_{\left.N\right|_{\Omega_{2}}}(\boldsymbol{\mu})=\sum_{j=1}^{N_{2}} \gamma_{j}^{2} p_{h}^{2}\left(\boldsymbol{\mu}_{2}^{j}\right)+\epsilon p_{H}^{2}(\boldsymbol{\mu}) .
\end{gathered}
$$

Note that the stresses associated with these functions are continuous across $\Gamma_{12}$, whereas the velocities are not. The continuity of velocities is imposed by solving the original Stokes problem on the whole domain, by using the Galerkin method on the spaces

$$
Y_{N_{1}}=\operatorname{span}\left\{\mathbf{u}_{h}^{1}\left(\boldsymbol{\mu}_{1}^{j}\right), \mathbf{v}_{h}^{1}\left(\boldsymbol{\mu}_{1}^{j}\right), \mathbf{u}_{H}^{1}(\boldsymbol{\mu}), \mathbf{v}_{H}^{1}(\boldsymbol{\mu}), j=1, \cdots, N_{1}\right\}
$$




$$
\begin{gathered}
Y_{N_{2}}=\operatorname{span}\left\{\mathbf{u}_{h}^{2}\left(\boldsymbol{\mu}_{2}^{j}\right), \mathbf{v}_{h}^{2}\left(\boldsymbol{\mu}_{2}^{j}\right), \mathbf{u}_{H}^{2}(\boldsymbol{\mu}), \mathbf{v}_{H}^{2}(\boldsymbol{\mu}), j=1, \cdots, N_{2}\right\}, \\
M_{N_{1}}=\operatorname{span}\left\{p_{h}^{1}\left(\boldsymbol{\mu}_{1}^{j}\right), p_{H}^{1}(\boldsymbol{\mu}), j=1, \cdots, N_{1}\right\}, \\
M_{N_{2}}=\operatorname{span}\left\{p_{h}^{2}\left(\boldsymbol{\mu}_{2}^{j}\right), p_{H}^{2}(\boldsymbol{\mu}), j=1, \cdots, N_{2}\right\}
\end{gathered}
$$

and using Lagrange multipliers.

More precisely we set

$$
\mathcal{L}(\mathbf{v}, \boldsymbol{\psi})=\int_{\Gamma_{12}} \llbracket \mathbf{v} \rrbracket \boldsymbol{\psi} d s, \forall \mathbf{v} \in Y_{N_{1}} \times Y_{N_{2}}, \forall \boldsymbol{\psi} \in W_{12}
$$

where $\llbracket \mathbf{v} \rrbracket=\mathbf{v}^{1}-\mathbf{v}^{2}$ denotes the jump of $\mathbf{v}$ across $\Gamma_{12}$ and $W_{12}$ is a low order polynomial space defined on $\Gamma_{12}$.

Then we consider the problem: find $\mathbf{u}_{N}(\boldsymbol{\mu}) \in Y_{N_{1}} \times Y_{N_{2}}, p_{N}(\boldsymbol{\mu}) \in M_{N_{1}} \times$ $M_{N_{2}}, \boldsymbol{\lambda}_{N} \in W_{12}$ such that

$$
\begin{cases}\mathcal{A}\left(\mathbf{u}_{N}(\boldsymbol{\mu}), \mathbf{w}, \boldsymbol{\mu}\right)+\mathcal{B}\left(\mathbf{w}, p_{N}(\boldsymbol{\mu}), \boldsymbol{\mu}\right)+\mathcal{L}\left(\mathbf{w}, \boldsymbol{\lambda}_{N}\right)=\mathcal{F}(\mathbf{w}, \boldsymbol{\mu}) & \forall \mathbf{w} \in Y_{N_{1}} \times Y_{N_{2}}, \\ \mathcal{B}\left(\mathbf{u}_{N}(\boldsymbol{\mu}), q, \boldsymbol{\mu}\right)=0 & \forall q \in M_{N_{1}} \times M_{N_{2}}, \\ \mathcal{L}\left(\mathbf{u}_{N}(\boldsymbol{\mu}), \boldsymbol{\psi}\right)=0 & \forall \boldsymbol{\psi} \in W_{12} .\end{cases}
$$

where $\boldsymbol{\lambda}_{N}$ represents the normal stresses on the internal inteface.

In general, if the velocities are approximated with piecewise continuous polynomial of order $k$ and pressures with polynomials of order $k-1$, then the normal stresses are polynomials of degree $k-1$. For this reason, the space of the Lagrange multipliers has been chosen as $W_{12}=\mathbb{P}^{k-1}\left(\Gamma_{12}\right)$.

The problem (27) can be written in compact form as:

$$
\left(\begin{array}{cc}
S & L \\
L^{T} & 0
\end{array}\right) \cdot\left(\begin{array}{l}
\boldsymbol{U} \\
\boldsymbol{\lambda}
\end{array}\right)=\left(\begin{array}{c}
F \\
0
\end{array}\right)
$$

where:

$S=\left(\begin{array}{cccc}A^{1} & 0 & B^{1} & 0 \\ 0 & A^{2} & 0 & B^{2} \\ B^{1} & 0 & 0 & 0 \\ 0 & B^{2} & 0 & 0\end{array}\right), L=\left(\begin{array}{c}L_{12}^{1} \\ -L_{12}^{2}\end{array}\right), F=\left(\begin{array}{c}F^{1} \\ F^{2}\end{array}\right), \boldsymbol{U}=\left(\begin{array}{c}\mathbf{u}_{N}^{1} \\ \mathbf{u}_{N}^{2} \\ \eta \\ \delta \\ \boldsymbol{p}_{N}^{1} \\ \boldsymbol{p}_{N}^{2} \\ \epsilon\end{array}\right), \boldsymbol{\lambda}=\left(\begin{array}{c}\lambda_{1} \\ \vdots \\ \lambda_{Q_{12}}\end{array}\right)$ 


$$
\begin{gathered}
A_{i j}^{k}=\mathcal{A}\left(\mathbf{w}_{i}^{k}, \mathbf{w}_{j}^{k}, \boldsymbol{\mu}_{k}\right), \quad B_{i l}^{k}=\mathcal{B}\left(\mathbf{w}_{i}^{k}, p_{l}^{k}, \boldsymbol{\mu}_{k}\right), \quad F_{j}^{k}=\mathcal{F}\left(\mathbf{w}_{j}^{k}, \boldsymbol{\mu}_{k}, \boldsymbol{\sigma}_{n}^{k}\right), \\
\left(L_{12}^{k}\right)_{q i}=\int_{\Gamma_{12}} \mathbf{w}_{i}^{k} \boldsymbol{\psi}_{q} d s, \quad \mathbf{w}_{i}, \mathbf{w}_{j} \in Y_{N_{k}}, \quad p_{l} \in M_{N_{k}}, \quad \boldsymbol{\psi}_{q} \in W_{12}, \\
\mathbf{u}_{N}^{1}=\left(\begin{array}{c}
\alpha_{1}^{1} \\
\vdots \\
\alpha_{N_{1}}^{1} \\
\beta_{1}^{1} \\
\vdots \\
\beta_{N_{1}}^{1}
\end{array}\right) \mathbf{u}_{N}^{2}=\left(\begin{array}{c}
\alpha_{1}^{2} \\
\vdots \\
\alpha_{N_{2}}^{2} \\
\beta_{1}^{2} \\
\vdots \\
\beta_{N_{2}}^{2}
\end{array}\right) \boldsymbol{p}_{N}^{1}=\left(\begin{array}{c}
\gamma_{1}^{1} \\
\vdots \\
\gamma_{N_{1}}^{1}
\end{array}\right) \boldsymbol{p}_{N}^{2}=\left(\begin{array}{c}
\gamma_{1}^{2} \\
\vdots \\
\gamma_{N_{2}}^{2}
\end{array}\right) \\
1 \leq i, j \leq 2 N_{k}+2, \quad 1 \leq l \leq N_{k}+1, \quad k=1,2, \quad 1 \leq q \leq 2 Q_{12},
\end{gathered}
$$

here $Q_{12}$ is the number of nodes considered on the interface $\Gamma_{12}$.

\subsection{Several subdomains with many internal interfaces}

In the most general case, we now consider a computational domain $\Omega$ parametrized through $\boldsymbol{\mu}=\left(\boldsymbol{\mu}_{1}, \cdots, \boldsymbol{\mu}_{r}\right)$ and decomposed in a non-overlapping union of $R$ subdomains $\Omega_{r}$, each one parametrized through the corresponding $\boldsymbol{\mu}_{r}$, see for example the domain shown in Figure 2. We make the assumption that each inflow boundary is included in one subdomain, each outflow boundary in another one, so that any other subdomain $\Omega_{r}$ has two internal interfaces. Our methodology can however be extended to more general domain partitions.

We still want to solve the problem (25) in a domain $\Omega$. The difference, with respect to what we have done in Section 5.1, is that now $\Omega$ is partitioned into $R$ subdomains $\Omega_{r}$ and each $\Omega_{r}$ is a deformation of a reference domain $\hat{\Omega}_{k(r)}$, for a suitable $k(r) \in\{1, \cdots, K\}, K$ being the number of reference shapes.

We focus now on computational strategy: as in the previous case of a portion with only two subdomains, see Section 5.1, we split it in two main stages. The offline stage involves the references blocks and it consists of the computation of independent reduced basis spaces and matrices (by using an accurate fine mesh $\mathcal{T}_{h}$ ). During the online stage, we use the results of the previous stage and we consider some proper computations and additional 
gluing conditions through the internal interfaces, in order to find a continuous global solution in the domain $\Omega$. With the term hybrid we want also underline that the online stage is not only depending on the dimensions $N_{i}$ of the reduced spaces, but it also includes some FE computation on a very coarse mesh $\mathcal{T}_{H}$ (however it does not involve any $\mathrm{FE}$ computations on $\mathcal{T}_{h}$, but only an interpolation procedure between the coarse and the fine discretization). The final goal is to have a fine solution at the cost of a coarse one, after proper pre-calculation (performed offline).

More precisely the following steps are performed in the offline stage.

- For every reference domain $\hat{\Omega}_{k(r)}$ we define the index $\operatorname{pos}(r) \in\{c$, in, out $\}$ and three reference "elements": $\hat{\Omega}_{k(r)}^{i n}$ ("inflow" element), $\hat{\Omega}_{k(r)}^{c}$ ("central" element), $\hat{\Omega}_{k(r)}^{\text {out }}$ ("outflow" element). In every "element", we formulate a Stokes problem in the form (16) : find $\left(\mathbf{u}\left(\boldsymbol{\mu}_{r}\right), p\left(\boldsymbol{\mu}_{r}\right)\right) \in$ $Y^{k(r)} \times M^{k(r)}$ such that

$$
\begin{cases}\mathcal{A}^{k(r)}\left(\mathbf{u}\left(\boldsymbol{\mu}_{r}\right), \mathbf{w}, \boldsymbol{\mu}_{r}\right)+\mathcal{B}^{k(r)}\left(\mathbf{w}, p\left(\boldsymbol{\mu}_{r}\right), \boldsymbol{\mu}_{r}\right)=\mathcal{F}^{k(r)}\left(\mathbf{w}, \boldsymbol{\mu}_{r}, \boldsymbol{\sigma}_{n}^{k(r)}\right) & \forall \mathbf{w} \in Y^{k(r),}, \\ \mathcal{B}^{k(r)}\left(\mathbf{u}\left(\boldsymbol{\mu}_{r}\right), q, \boldsymbol{\mu}_{r}\right)=0 & \forall q \in M^{k(r)} .\end{cases}
$$

These local Stokes problems are defined in each $\hat{\Omega}_{k(r)}^{p o s(k)}, r=1, \cdots, R$ and the corresponding boundary conditions, as well as the spaces $Y^{k(r)}$ and $M^{k(r)}$, have been set as proposed in Section 3 .

- We solve problems (30) for several values of the (sampling) parameter $\boldsymbol{\mu}_{r}$ and we define the corresponding reduces spaces. More precisely, since $k(r) \in\{1, \cdots, K\}$ and $\operatorname{pos}(r) \in\{c$, in,$o u t\}$, we define $3 K$ spaces:

$$
\begin{gathered}
Y_{k(r)}^{p o s(r)}=\operatorname{span}\left\{\mathbf{u}_{h}^{k(r)}\left(\boldsymbol{\mu}_{r}^{i}\right), \mathbf{v}_{h}^{k(r)}\left(\boldsymbol{\mu}_{r}^{i}\right), i=1, \ldots, N_{k(r)}^{\text {pos }(r)}\right\}, \\
M_{k(r)}^{\text {pos }(r)}=\operatorname{span}\left\{p_{h}^{k(r)}\left(\boldsymbol{\mu}_{r}^{i}\right), i=1, \ldots, N_{k(r)}^{\text {pos }(r)}\right\}
\end{gathered}
$$

where $\boldsymbol{\mu}_{r}^{i}$ are the samples chosen by the greedy algorithm in $\hat{\Omega}_{k(r)}$; $N_{k(r)}^{\text {pos }(r)}$ represents the number of these samples and also of the precomputed basis functions: in general it may be different for each reference domain and for each Neumann boundary condition set. With this strategy, we can have proper spaces for each possible position of the corresponding deformed subdomain $\Omega_{r}$ (inflow, central, outflow element) on $\Omega$. 
In the online stage, for every given new parameter $\boldsymbol{\mu}=\left(\boldsymbol{\mu}_{1}, \cdots, \boldsymbol{\mu}_{r}\right)$ and for every generic combination of the deformed subdomains $\Omega_{r} \subset \Omega, r=1, \cdots, R$, we have the following steps.

- For every $\Omega_{r}$, we select the proper corresponding precomputed reduced basis spaces, depending on $k(r)$ and on the "position" of $\Omega_{r}$ in $\Omega$ (inflow, central or outflow element). We denote with $\mathbf{u}_{h}^{\mathbf{r}}\left(\boldsymbol{\mu}_{r}^{i}\right), \mathbf{v}_{h}^{\mathbf{r}}\left(\boldsymbol{\mu}_{r}^{i}\right)$ and $p_{h}^{\mathbf{r}}\left(\boldsymbol{\mu}_{r}^{i}\right)$ the reduced basis functions (snapshots) of the corresponding spaces $Y_{k(r)}^{\text {pos }(r)}$ and $M_{k(r)}^{\text {pos(r) }}$ computed in the domain $\hat{\Omega}_{k(r)}$, where the index $\mathbf{r}$ is a couple of integers: $k(r)$ and the position index $\operatorname{pos}(r)$, $\mathbf{r}=(k(r), \operatorname{pos}(r))$.

- A Finite Element solution $\mathbf{u}_{H}(\boldsymbol{\mu}), p_{H}(\boldsymbol{\mu})$ of the Stokes problem (25) is computed in a fast way by using a coarse mesh $\mathcal{T}_{H}$ on the whole domain $\Omega^{1}$. Together with the associated supremizer solution $\mathbf{v}_{H}(\boldsymbol{\mu})$, we restrict these functions to each subdomain $\Omega_{r}$, then we map them in the corresponding reference domain $\hat{\Omega}_{k(r)}$. We denote with $\mathbf{u}_{H}^{r}(\boldsymbol{\mu})$, $\mathbf{v}_{H}^{r}(\boldsymbol{\mu}), p_{H}^{r}(\boldsymbol{\mu})$ the interpolation of these coarse solutions on the fine mesh $\mathcal{T}_{h}$. These functions are obviously continuous along the internal interfaces and they bring important physical information.

- We define in $\hat{\Omega}_{k(r)}$ the following spaces:

$$
\begin{aligned}
Y^{\mathbf{r}} & =\operatorname{span}\left\{\mathbf{u}_{h}^{\mathbf{r}}\left(\boldsymbol{\mu}_{r}^{i}\right), \mathbf{v}_{h}^{\mathbf{r}}\left(\boldsymbol{\mu}_{r}^{i}\right), \mathbf{u}_{H}^{r}(\boldsymbol{\mu}), \mathbf{v}_{H}^{r}(\boldsymbol{\mu}), i=1, \ldots, N^{\mathbf{r}}\right\}, \\
M^{\mathbf{r}} & =\operatorname{span}\left\{p_{h}^{\mathbf{r}}\left(\boldsymbol{\mu}_{r}^{i}\right), p_{H}^{r}(\boldsymbol{\mu}), i=1, \ldots, N^{\mathbf{r}}\right\},
\end{aligned}
$$

where $\mathbf{r}=(k(r), \operatorname{pos}(r)), r=1, \cdots, R, k \in\{1, \cdots, K\}, \operatorname{pos}(r) \in$ $\{$ in $, c, o u t\}$ and $N^{\mathbf{r}}=N_{k(r)}^{p o s(r)}$.

- Recalling that $\boldsymbol{\mu}$ is the selection of the parameters $\boldsymbol{\mu}_{r}, r=1, \cdots, R$ which define the computational domain $\bar{\Omega}=\bigcup_{r=1}^{R} \overline{\Omega_{r}}$, the global reduced basis "hybrid" solution is defined by a suitable local linear combination of the precomputed functions that define $\left(\mathbf{u}_{N}\left(\boldsymbol{\mu}_{r}\right), p_{N}\left(\boldsymbol{\mu}_{r}\right)\right)$ on each subdomain $\Omega_{r}$ as follows, for $r=1, \cdots, R$ :

\footnotetext{
${ }^{1}$ The coarse solution is inexpensive from a computational point of view and also from practical point of view since it is computed on a combination and repetition of only reference subdomains, which are easily constructed by translation starting by the reference sub-blocks.
} 


$$
\begin{gathered}
\left.\mathbf{u}_{N}\right|_{\Omega_{r}}(\boldsymbol{\mu})=\sum_{i=1}^{N^{\mathbf{r}}} \alpha_{i}^{r} \mathbf{u}_{h}^{\mathbf{r}}\left(\boldsymbol{\mu}_{r}^{i}\right)+\sum_{i=1}^{N^{\mathbf{r}}} \beta_{i}^{r} \mathbf{v}_{h}^{\mathbf{r}}\left(\boldsymbol{\mu}_{r}^{i}\right)+\eta \mathbf{u}_{H}^{r}(\boldsymbol{\mu})+\delta \mathbf{v}_{H}^{r}(\boldsymbol{\mu}) \\
\left.p_{N}\right|_{\Omega_{r}}(\boldsymbol{\mu})=\sum_{i=1}^{N^{\mathbf{r}}} \gamma_{i}^{r} p_{i}^{\mathbf{r}}\left(\boldsymbol{\mu}_{r}^{i}\right)+\epsilon p_{H}^{r}(\boldsymbol{\mu})
\end{gathered}
$$

The coefficients $\alpha_{j}^{r}, \beta_{j}^{r}, \gamma_{j}^{r}$ and $\eta, \delta, \epsilon$ are determined by solving the original problem (25) on $\Omega$ by using the Galerkin method on the reduced spaces $Y^{\boldsymbol{r}}$ and $M^{\boldsymbol{r}}$. Since the spaces $Y^{\boldsymbol{r}}$ are made of discontinuous velocities, their jumps across the interfaces are minimized through the use of Lagrange multipliers. With this aim, we define $\forall \boldsymbol{\psi} \in W_{m l}, m, l \in\{1, \cdots, R\}$

$$
\mathcal{L}^{m l}\left(\mathbf{u}_{N}(\boldsymbol{\mu}), \boldsymbol{\psi}\right)=\int_{\Gamma_{m l}}\left(\left.\mathbf{u}_{N}\right|_{\Omega_{m}}(\boldsymbol{\mu})-\left.\mathbf{u}_{N}\right|_{\Omega_{l}}(\boldsymbol{\mu})\right) \boldsymbol{\psi} d s
$$

where $\Gamma_{m l}$ is the interface between two adjacent subdomains denoted with the indices $m$ and $l$ respectively and $W_{m l}$ is a low order polynomial space defined on this interface, see $[5 ; 21]$. A basis for $W_{m l}$ is provided by the characteristic Lagrange polynomials $\boldsymbol{\psi}_{q}, q=1, \cdots, Q_{m l}$ associated with the $Q_{m l}$ nodes of $\Gamma_{m l}$.

If we suppose that $\Omega$ has $R-1$ internal interfaces, $\Gamma_{i i+1}, i=1, \cdots, R-1$, we therefore solve the following problem (which generalizes problem (27) that was set for the two subdomains case): find $\mathbf{u}_{N}(\boldsymbol{\mu}) \in Y^{\mathbf{1}} \times$ $\cdots \times Y^{\boldsymbol{R}}, p_{N}(\boldsymbol{\mu}) \in M^{\mathbf{1}} \times \cdots \times M^{\boldsymbol{R}}, \boldsymbol{\lambda}_{N} \in W_{i i+1}, i=1, \cdots, R-1$, such that

$$
\left\{\begin{array}{lr}
\mathcal{A}\left(\mathbf{u}_{N}(\boldsymbol{\mu}), \mathbf{w}, \boldsymbol{\mu}\right)+\mathcal{B}\left(\mathbf{w}, p_{N}(\boldsymbol{\mu}), \boldsymbol{\mu}\right)+\sum_{i=1}^{R-1} & \mathcal{L}^{i i+1}\left(\mathbf{w}, \boldsymbol{\lambda}_{N}\right)=\mathcal{F}(\mathbf{w}, \boldsymbol{\mu}) \\
& \forall \mathbf{w} \in Y^{\mathbf{1}} \times \cdots \times Y^{\boldsymbol{R}}, \\
\mathcal{B}\left(\mathbf{u}_{N}(\boldsymbol{\mu}), q, \boldsymbol{\mu}\right)=0 & \forall q \in M^{\mathbf{1}} \times \cdots \times M^{\boldsymbol{R}}, \\
\mathcal{L}^{i i+1}\left(\mathbf{u}_{N}(\boldsymbol{\mu}), \boldsymbol{\psi}\right)=0 & i=1, \ldots, R-1, \forall \boldsymbol{\psi} \in W_{i i+1} .
\end{array}\right.
$$

As before, on each interface $\boldsymbol{\lambda}_{N}$ represents the normal stresses. In this case the subdomains on $\Omega$ are "consecutives", for every $\Omega_{r}, r=$ $2, \ldots, R-1$, there are just two internal interfaces $\Gamma_{r-1 r}$ and $\Gamma_{r r+1}$, but 
in the most general case, we have to consider in problem (34) every internal interface $\Gamma_{m l}$ and every corresponding bilinear form $\mathcal{L}^{m l}(\cdot, \cdot)$. As already mentioned in Section 2, in case of null force term, the local Stokes problem for the central domains might generate a null solution. In this particular case, the global solution will coincide with the interpolation of the coarse one. However, every tests has been carried out by imposing a non-zero force term.

\subsection{Computational strategy}

In order to illustrate and build the final linear system that has to be solved, we consider another example made up by three subdomains:

$$
\begin{aligned}
& \bar{\Omega}(\boldsymbol{\mu})=\overline{\Omega_{1}} \cup \overline{\Omega_{2}} \cup \overline{\Omega_{3}}=\overline{T_{\boldsymbol{\mu}_{1}}^{1}\left(\hat{\Omega}_{1}\right)} \cup \overline{T_{\boldsymbol{\mu}_{2}}^{1}\left(\hat{\Omega}_{1}\right)} \cup \overline{T_{\boldsymbol{\mu}_{3}}^{2}\left(\hat{\Omega}_{2}\right)}, \quad \Omega_{1} \cap \Omega_{2} \cap \Omega_{3}=\emptyset, \\
& \Gamma_{12}=\bar{\Omega}_{1} \cap \bar{\Omega}_{2}, \quad \Gamma_{23}=\bar{\Omega}_{2} \cap \bar{\Omega}_{3} .
\end{aligned}
$$

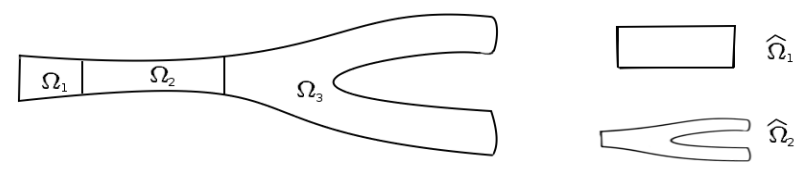

Figure 3: Geometrical scheme for a domain composed by $\mathrm{R}=3$ deformations of $\mathrm{K}=2$ reference domains.

In this case the three subdomains can be obtained as deformations of two different reference domains $\hat{\Omega}_{1}$ and $\hat{\Omega}_{2}$.

As described in the section 5.2 (offline stage), we have three local problems in the form (30), and we build independently (i.e. in parallel computations) the three couples of reduced basis spaces $Y_{1}^{\text {in }}, M_{1}^{\text {in }}, Y_{1}^{c}, M_{1}^{c}, Y_{2}^{\text {out }}, M_{2}^{\text {out }}{ }^{2}{ }^{2}$ In the online stage, for the parameter $\boldsymbol{\mu}=\left(\boldsymbol{\mu}_{1}, \boldsymbol{\mu}_{2}, \boldsymbol{\mu}_{3}\right)$, we compute the coarse global solution, we interpolate it in the fine mesh and we add the restrictions to the reduced spaces. The interpolation process of the coarse mesh on the finer one is still a reasonable operation in terms of online computational cost, but as shown in the numerical tests (next section), it is always

\footnotetext{
${ }^{2}$ In order to solve many other possible configurations in the online step, we may have to compute also the spaces $Y_{1}^{\text {out }}, M_{1}^{\text {out }}, Y_{2}^{\text {in }}, M_{2}^{\text {in }}, Y_{2}^{c}, M_{2}^{c}$.
} 
substantially less expensive than computing a true fine finite element solution.

We define:

$$
\begin{aligned}
& Y^{\mathbf{1}}=\operatorname{span}\left\{\left.\mathbf{u}_{H}\right|_{\hat{\Omega}_{1}},\left.\mathbf{v}_{H}\right|_{\hat{\Omega}_{1}}, \mathbf{u}_{h}^{\mathbf{1}}\left(\boldsymbol{\mu}_{1}^{i}\right), \mathbf{v}_{h}^{\mathbf{1}}\left(\boldsymbol{\mu}_{1}^{i}\right), i=1, \ldots, N^{\mathbf{1}}\right\}, \\
& M^{\mathbf{1}}=\operatorname{span}\left\{\left.p_{H}\right|_{\hat{\Omega}_{1}}, p_{h}^{\mathbf{1}}\left(\boldsymbol{\mu}_{1}^{i}\right), i=1, \ldots, N^{\mathbf{1}}\right\}, \\
& Y^{\mathbf{2}}=\operatorname{span}\left\{\left.\mathbf{u}_{H}\right|_{\hat{\Omega}_{1}},\left.\mathbf{v}_{H}\right|_{\hat{\Omega}_{1}}, \mathbf{u}_{h}^{\mathbf{2}}\left(\boldsymbol{\mu}_{2}^{i}\right), \mathbf{v}_{h}^{\mathbf{2}}\left(\boldsymbol{\mu}_{2}^{i}\right), i=1, \ldots, N^{\mathbf{2}}\right\}, \\
& M^{\mathbf{2}}=\operatorname{span}\left\{\left.p_{H}\right|_{\hat{\Omega}_{1}}, p_{h}^{\mathbf{2}}\left(\boldsymbol{\mu}_{2}^{i}\right), i=1, \ldots, N^{\mathbf{2}}\right\}, \\
& Y^{\mathbf{3}}=\operatorname{span}\left\{\left.\mathbf{u}_{H}\right|_{\hat{\Omega}_{2}},\left.\mathbf{v}_{H}\right|_{\hat{\Omega}_{2}}, \mathbf{u}_{h}^{\mathbf{3}}\left(\boldsymbol{\mu}_{3}^{i}\right), \mathbf{v}_{h}^{\mathbf{3}}\left(\boldsymbol{\mu}_{3}^{i}\right), i=1, \ldots, N^{\mathbf{3}}\right\}, \\
& M^{\mathbf{3}}=\operatorname{span}\left\{\left.p_{H}\right|_{\hat{\Omega}_{2}}, p_{h}^{\mathbf{3}}\left(\boldsymbol{\mu}_{3}^{i}\right), i=1, \ldots, N^{\mathbf{3}}\right\} .
\end{aligned}
$$

Here the index $\mathbf{r}=\mathbf{1}$ refers to $\operatorname{pos}(1)=i n$ and $k(1)=1$, the index $\mathbf{r}=\mathbf{2}$ to $\operatorname{pos}(2)=c$ and $k(2)=1$ and the index $\mathbf{r}=\mathbf{3}$ to $\operatorname{pos}(3)=$ out and $k(3)=2$. The final linear system of the reduced basis hybrid problem includes the matrices of the three problems defined in the offline stage (that correspond to the matrices $A^{r}$ and $B^{r}$ and the vectors $F^{r}, r=1,2,3$ defined in (29)) and two matching conditions (corresponding to the two internal interfaces) described by (33) (involving the matrices $L_{l m}^{r}, l=1,2, m=2,3$ defined in $(36))$ :

$$
\left(\begin{array}{cccccccc}
A^{1} & 0 & 0 & B^{1} & 0 & 0 & L_{12}^{1} & 0 \\
0 & A^{2} & 0 & 0 & B^{2} & 0 & -L_{12}^{2} & L_{23}^{2} \\
0 & 0 & A^{3} & 0 & 0 & B^{3} & 0 & -€_{23}^{3} \\
B^{1 T} & 0 & 0 & 0 & 0 & 0 & 0 & 0 \\
0 & B^{2 T} & 0 & 0 & 0 & 0 & 0 & 0 \\
0 & 0 & B^{3 T} & 0 & 0 & 0 & 0 & 0 \\
L_{12}^{1 T} & -L_{12}^{2 T} & 0 & 0 & 0 & 0 & 0 & 0 \\
0 & L_{23}^{2 T} & -L_{23}^{3 T} & 0 & 0 & 0 & 0 & 0
\end{array}\right) \cdot\left(\begin{array}{c}
\boldsymbol{u}_{N} \\
\\
\boldsymbol{p}_{N} \\
\lambda_{1} \\
\lambda_{2}
\end{array}\right)=\left(\begin{array}{c}
F^{1} \\
F^{2} \\
F^{3} \\
0 \\
0 \\
0 \\
0 \\
0
\end{array}\right)
$$

where 
$\mathbf{u}_{N}=\left(\begin{array}{c}\boldsymbol{\alpha}^{1} \\ \boldsymbol{\beta}^{1} \\ \boldsymbol{\alpha}^{2} \\ \boldsymbol{\beta}^{2} \\ \boldsymbol{\alpha}^{3} \\ \boldsymbol{\beta}^{3} \\ \eta \\ \delta\end{array}\right) \boldsymbol{p}_{N}=\left(\begin{array}{c}\gamma^{1} \\ \gamma^{2} \\ \boldsymbol{\gamma}^{3} \\ \epsilon\end{array}\right) \boldsymbol{\alpha}^{i}=\left(\begin{array}{c}\alpha_{1}^{i} \\ \vdots \\ \alpha_{N_{i}}^{i}\end{array}\right) \boldsymbol{\beta}^{i}=\left(\begin{array}{c}\beta_{1}^{i} \\ \vdots \\ \beta_{N_{i}}^{i}\end{array}\right) \boldsymbol{\gamma}^{i}=\left(\begin{array}{c}\gamma_{1}^{i} \\ \vdots \\ \gamma_{N_{i}}^{i}\end{array}\right) i=1,2,3$,
$\left(L_{m l}^{m}\right)_{q i}=\int_{\Gamma_{m l}} \mathbf{u}_{h}^{m}\left(\boldsymbol{\mu}_{m}^{i}\right) \boldsymbol{\psi}_{q} d s, \quad m, l \in\{1, \cdots, R\}, 1 \leq q \leq 2 Q_{m l}, 1 \leq i \leq 2 N^{\boldsymbol{m}}$.

We note that for the example at hand reported schematically in Figure 3, the dimension of the linear system is determined by two quantities: the dimensions of the reduced basis spaces, $N^{\mathbf{1}}, N^{\mathbf{2}}$ and $N^{\mathbf{3}}$, the corresponding dimensions of $A^{1}, A^{2}, A^{3}$ (respectively $2 N^{\mathbf{1}} \times 2 N^{\mathbf{1}}, 2 N^{\mathbf{2}} \times 2 N^{\mathbf{2}}$ and $2 N^{\mathbf{3}} \times 2 N^{\mathbf{3}}$ ) and $B^{1}, B^{2}, B^{3}$ (respectively $2 N^{\mathbf{1}} \times N^{\mathbf{1}}, 2 N^{\mathbf{2}} \times N^{\mathbf{2}}$ and $2 N^{\mathbf{3}} \times N^{\mathbf{3}}$ ); the number of nodes $K_{r m}$ on the internal interfaces $\Gamma_{r m}$ affects the dimension of $L_{12}^{1}, L_{12}^{2}$, $L_{23}^{2}$ and $L_{23}^{3}$ (given respectively by $2 N^{\mathbf{1}} \times K_{12}, 2 N^{\mathbf{2}} \times K_{12}, 2 N^{\mathbf{2}} \times K_{23}$ and $\left.2 N^{3} \times K_{23}\right)$. Without any further assumption on the basis for the velocity and the pressure spaces, in order to match the $n Q_{m l}$ Lagrange multipliers equations with the $N^{m}$ basis functions of each subdomain,we have always imposed $N^{m}>Q_{m l}$.

\section{Transfinite maps}

In this section we recall the method to generate parametrized transfinite maps as proposed in [16] and, more recently, in [18], which can been seen as a generalization of the Gordon-Hall transfinite interpolation approach for quadrilaterals [11]. As seen above, these maps can be used to deform the subdomains of the computational domain in which we want to solve the local Stokes problem.

A transfinite map induces non-affine geometrical parametrization and, as presented in Section 3, on this map the empirical interpolation method is applied to recover the affinity of the linear and bilinear forms of the studied 
problem. Other options to parametrize the domains by a global map are proposed in $[14 ; 19 ; 22 ; 23]$.

We assume a general domain $\Omega$ and a general reference domain $\hat{\Omega}$, we suppose that both are curved polygons with the same number $n$ of curved edges. $\Gamma_{i}$ denotes the generic edge in $\Omega, \hat{\Gamma}_{i}$ denotes the corresponding edge in $\hat{\Omega}$; the edges are numbered clockwise.

We associate a weight function $\varphi_{i}$ to each side $\hat{\Gamma}_{i}, i=1, \ldots, n$ of a reference domain $\hat{\Omega}$ with $n$-side. To define $\varphi_{i}$ on $\hat{\Gamma}_{i}$, we impose $\varphi_{i}=1$ on $\hat{\Gamma}_{i}$, and solve the Laplace problem:

$$
\Delta \varphi_{i}=0 \quad \text { in } \hat{\Omega}
$$

with homogeneous Neumann boundary conditions on the two sides of $\hat{\Omega}$ adjacent to $\hat{\Gamma}_{i}$, and homogeneous Dirichlet boundary conditions on the remaining sides (Figure 4).

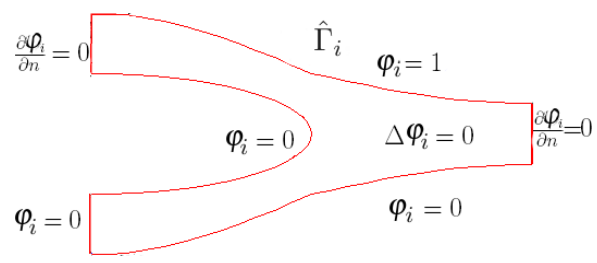

Figure 4: Graphical representation of the boundary conditions for weight functions problem in a reference bifurcated domain.

To define the transfinite map on a general reference domain, we also need the projection of the internal part onto each side $\hat{\Gamma}_{i}$. We compute the projection function $\pi_{i}$ onto the side $\hat{\Gamma}_{i}$, by solving the Laplace problem:

$$
\Delta \pi_{i}=0 \quad \text { in } \hat{\Omega}
$$

with a Dirichlet boundary condition along $\hat{\Gamma}_{i}$ being a linear function of the arc-length $t$ ranging 0 to 1 . On the sides adjacent to $\hat{\Gamma}_{i}$ we set $\pi_{i}$ equal to either 0 or 1 , and on the remaining sides we impose homogeneous Neumann boundary conditions (Figure 5).

For each side of the reference domain, we have associated one weight function and one projection function by solving the problems (37) and (38) respectively (Figure 8 ). For a domain with $n$ sides, we have to solve $2 n$ elliptic 


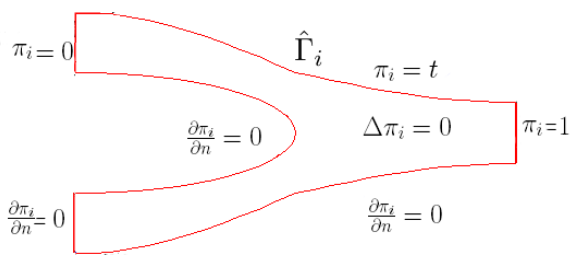

Figure 5: Graphical representation of the boundary condition for the projection functions problem in a reference bifurcated domain.

problems, however these computations are independent of the deformation (and of the parameter) and can be included in the offline stage of the reduced basis method.
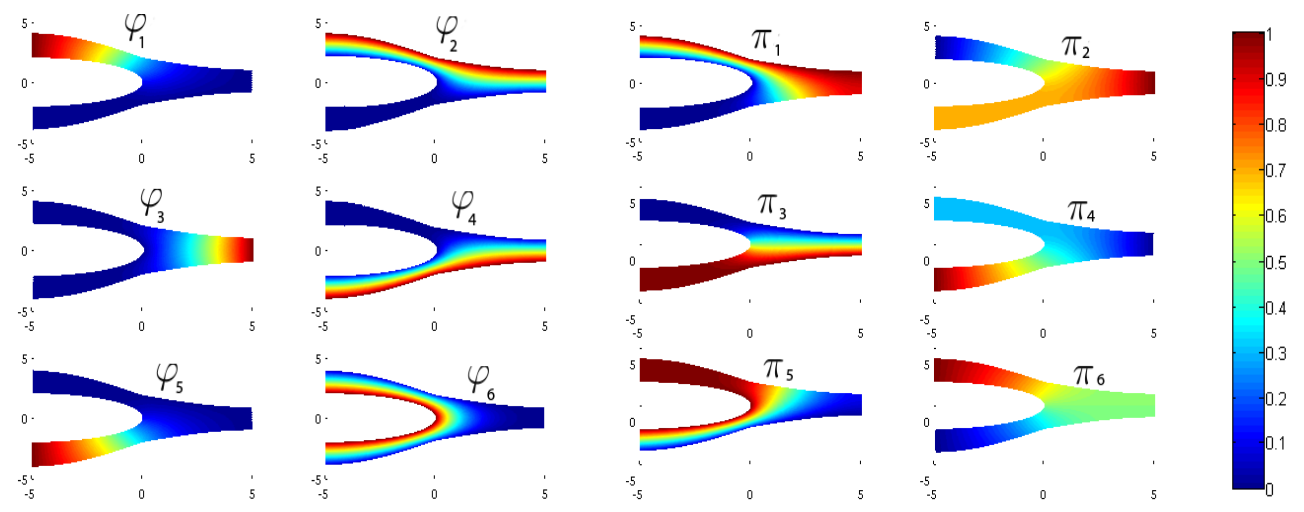

Figure 6: Weight functions $\varphi_{i}$, solutions of (37) (on the left) and projection functions $\pi_{i}$, solutions of (38)(on the right) for a reference bifurcation; $1 \leq i \leq 6$.

We assume that each edge is parametrized through the parameter $\boldsymbol{\mu} \in \mathcal{D}$ by a bijective map $\psi_{i}$ from $[0,1] \times \mathcal{D}$ into $\Gamma_{i}$, so that $\psi_{i}(1, \boldsymbol{\mu})=x_{i}$, where $x_{i}$ denotes the vertex shared by $\Gamma_{i}$ and $\Gamma_{i+1}$ and $\psi_{i}(0, \boldsymbol{\mu})=x_{i-1}$. We denote by $\hat{x}$ a generic point of the reference domain $\hat{\Omega}$ and by $x$ a generic point of the deformed domain $\Omega$. The transfinite map is then defined as follows:

$$
T(\hat{x}, \boldsymbol{\mu})=\sum_{i=1}^{n}\left\{\varphi_{i}(\hat{x}) \psi_{i}\left(\pi_{i}(\hat{x}), \boldsymbol{\mu}\right)-\varphi_{i}(\hat{x}) \varphi_{i+1}(\hat{x}) x_{i}\right\}
$$

We show the deformations of two domains, used as subdomain blocks for numerical tests of RBHM, obtained through the transfinite map. 
We consider a bifurcation described by three different parameters: $\mu_{1}$ represents the length of the bifurcation, $\mu_{2}$ the thickness and $\mu_{3}$ the span between the branches. Figure 6 shows the weight functions $\varphi_{i}$ and projection functions $\pi_{i}$ for the reference bifurcation, while Figure 7 shows some possible examples of bifurcation deformations obtained by applying the transformation (39).
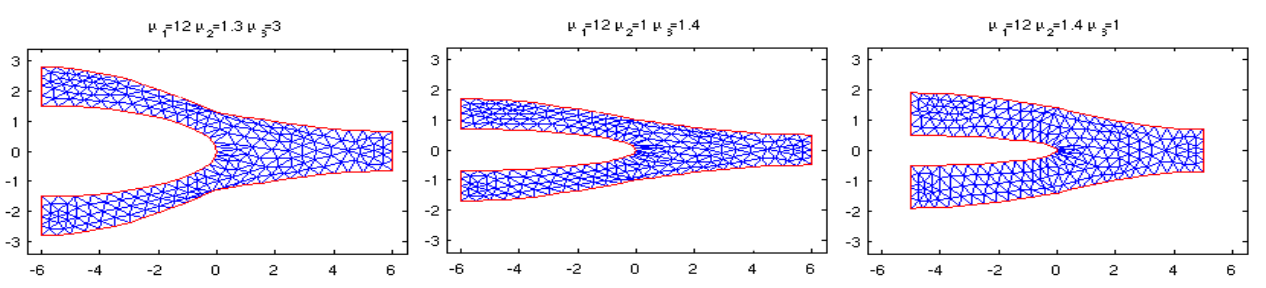

Figure 7: Different bifurcation deformations.

A deformed pipe, with parametrized upper and lower walls, is used to simulate a stenosis in an artery of the cardiovascular system. The parameters $\mu_{1}$ and $\mu_{2} \in[-1,1]$ represent the dilatation and the contraction of the pipe. Figure 8 shows the weight functions $\varphi_{i}$ and projection functions $\pi_{i}$ for the reference rectangle, while Figure 9 shows some possible pipe deformations.

The advantage of using the extended transfinite map in the context of a reduced basis method (as well as RBEM and RBHM), is that all the harmonic functions $\varphi_{i}$ and $\pi_{i}$ may be computed in the offline stage. In the online stage we need only to compute the inexpensive boundary functions $\psi_{i}\left(\pi_{i}(\hat{x}), \boldsymbol{\mu}\right)$ and perform the linear combination of the harmonic functions in (39) [11; 17].

\section{Numerical tests on 2D domains}

Numerical tests were carried out on different problems such as stenosis and bifurcation to solve Stokes ${ }^{3}$ flows with the reduced basis hybrid method using the non-affine transfinite map. Taylor-Hood Finite Element Method has been used to compute approximation basis functions, $\mathbb{P}_{2}$ elements for

\footnotetext{
${ }^{3}$ Under the assumption we are considering low Reynolds numbers, with low average blood velocity and in mid-sized arteries, this is an acceptable approximation (for example in coronary arteries), [1;27].
} 

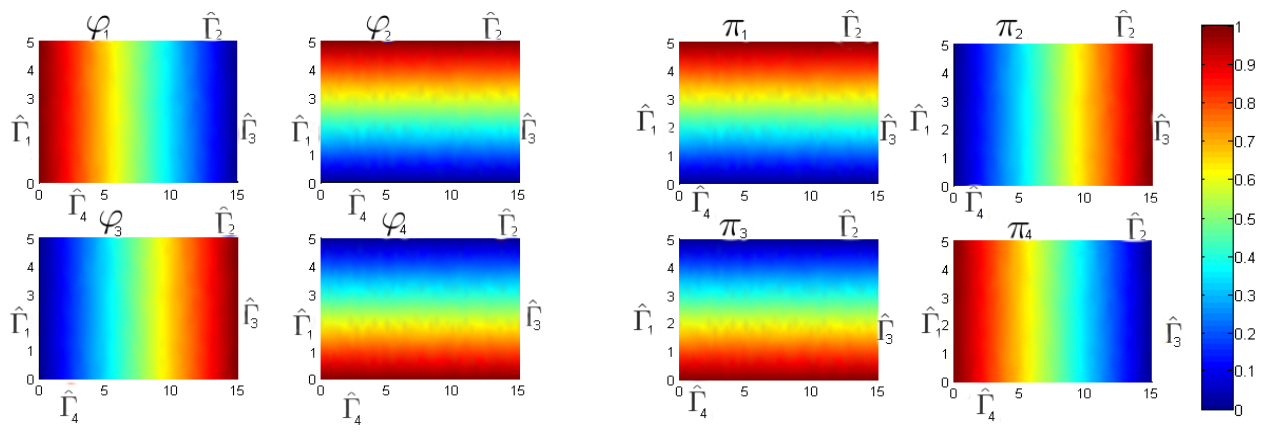

Figure 8: Weight functions $\varphi_{i}$, solutions of (37) (on the left) and projection functions $\pi_{i}$, solutions of (38)(on the right) for a reference rectangle; $1 \leq i \leq 4$.
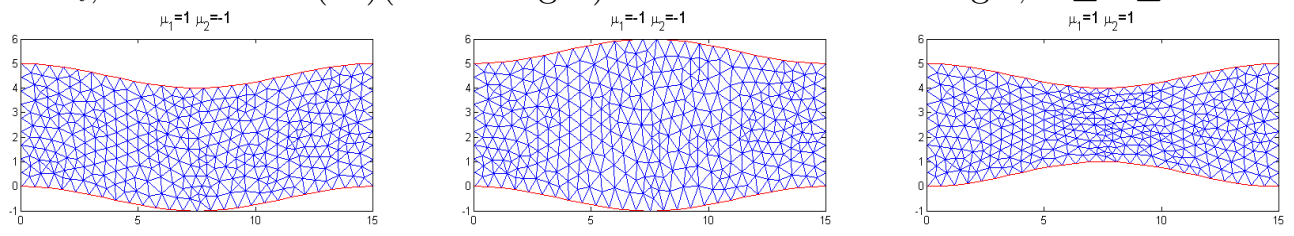

Figure 9: Different pipe deformations.

velocity and supremizer, $\mathbb{P}_{1}$ for pressure, respectively [30]. Consequently the Lagrange multipliers spaces is $\mathbb{P}_{1}\left(\Gamma_{m l}\right)$.

\subsection{A stenosed arterial vessel}

As first proposed test we model the blood flow through an artery occluded by stenoses [10].Every stenosis represents a subdomain and it has curved and parametrized walls.

In particular, the deformations of the single stenosis domain depend on two parameters: the amplitudes, $\mu_{1} \in[0,2]$ and $\mu_{2} \in[0,2]$, on the upper and lower walls representing two independent contractions, respectively.

The deformed single block domain $\Omega_{i}$ in Figure 10 is mapped starting from the straight reference pipe $\hat{\Omega}_{1}$ of length $L$ and height $D$ through a transfinite map.

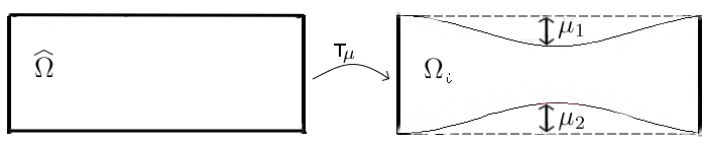

Figure 10: Geometrical scheme for the stenosis block.

The computational domain $\Omega$ for the model has been composed by three stenosed blocks so that $\bar{\Omega}=\cup_{i=1}^{3} \bar{\Omega}_{\boldsymbol{\mu}_{i}}$, as shown in Figure 11 . 


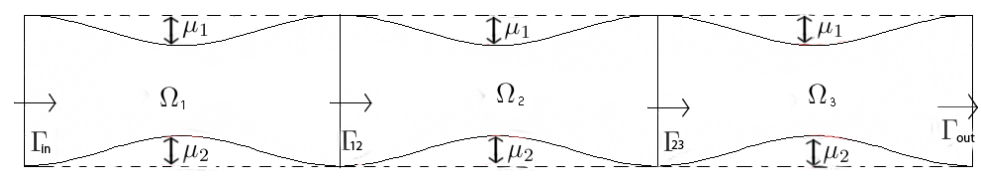

Figure 11: Geometrical scheme for the computational domain.

We consider a parametrized Stokes problem (23) for each subdomain, where the force field $\boldsymbol{f}$ is the gravity. For the inflow subdomain, we compute the reduced basis imposing zero Dirichlet condition on the wall, Neumann boundary conditions given by imposing $\boldsymbol{\sigma}_{n}=\boldsymbol{\sigma} \mathbf{n}=\nu \frac{\partial \mathbf{u}}{\partial \mathbf{n}}-p \mathbf{n}$ to be $\boldsymbol{\sigma}_{n}^{i n}=[1,0]^{T}$ on $\Gamma_{i n}$ and $\boldsymbol{\sigma}_{n}^{\text {out }}=\mathbf{0}$ on the internal interface $\Gamma_{12}$. For the outflow subdomain, we compute the reduced basis imposing zero Dirichlet condition on the wall, Neumann boundary conditions imposing $\boldsymbol{\sigma}_{n}^{i n}=\mathbf{0}$ on the internal interface $\Gamma_{23}$ and $\boldsymbol{\sigma}_{n}^{\text {out }}=[-1,0]^{T}$ on the outflow interface $\Gamma_{\text {out }}$. When we consider the internal subdomain, we impose zero Dirichlet condition on the walls and homogeneous Neumann boundary conditions on $\Gamma_{12}$ and $\Gamma_{23}$.

We apply the transfinite map to transform the problem in terms of reference coordinates.

By referring to a single stenosed block we expand each geometrical component in order to deal with an affine decomposition. We use the empirical interpolation to decompose the terms (8), (11) and (12). The maximum interpolation error is set to $\epsilon_{\text {tol }}^{E I M}=10^{-6}$.

By applying the offline stage of the reduced basis method to the single stenosis block, a set of $N=40$ combinations of parameters is selected by the greedy algorithm [31] using a tolerance $\epsilon_{\text {greedy }}=10^{-7}$.

Figure 12 shows the clustered distribution of these parameters used to store the basis functions [36]. Note that all the previous contributions concerning a posteriori error bounds are still valid in the single block [33].

Coarse and fine grids have been chosen in order to deal with respectively 200 and 1583 nodes in the whole domain $\Omega$, so that $H / h \approx 3$.

Figure 13 shows an example of a representative flow solution, found with the reduced basis hybrid method, for certain parameters combination $\left(\boldsymbol{\mu}_{1}=\right.$ $\left.(0.1,2), \boldsymbol{\mu}_{2}=(0.1,2), \boldsymbol{\mu}_{3}=(2,0.1)\right)$, to be compared with the finite element solution. The same comparison, regarding the pressure solutions, is shown in Figure 14. 


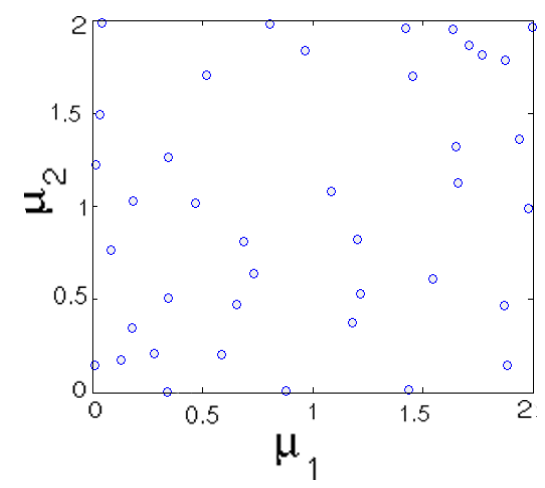

Figure 12: Parameter distribution representing the parameters combinations selected to generate the basis functions in a single block.

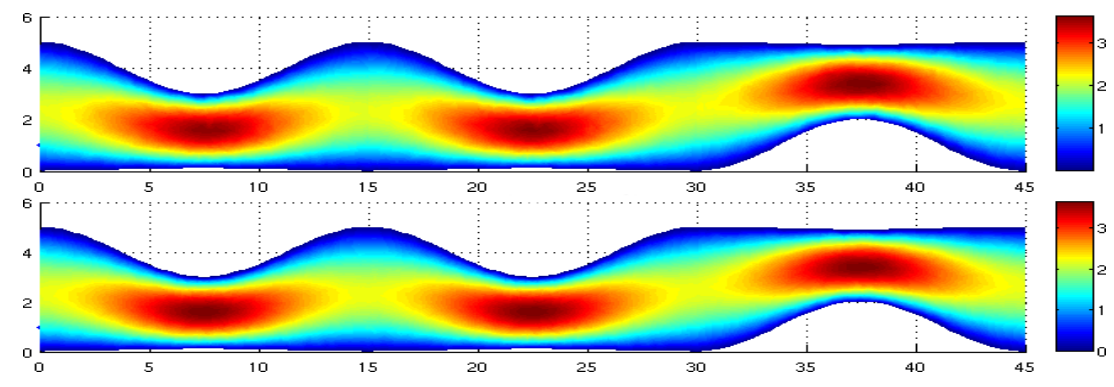

Figure 13: Velocity intensity $\left[\mathrm{ms}^{-1}\right]$ representative solutions using RBHM (with $N_{1}=$ $\left.N_{2}=N_{3}=10\right)$ (top) and solving a global FEM (bottom), $\left(\boldsymbol{\mu}_{1}=(0.1,2), \boldsymbol{\mu}_{2}=(0.1,2)\right.$, $\left.\boldsymbol{\mu}_{3}=(2,0.1)\right)$.

Figure 15 shows the reduction of the $H^{1}$ and $L^{2}$ relative errors, respectively, on velocity and pressure between the "truth" FE solution and the RB approximation. We plot the minimum, the maximum and the average of the errors obtained with a set of 1000 samples, increasing the number $N$ of basis functions.

Figures 16 and 17 show the pressure profiles on the internal interfaces $\Gamma_{12}$ and $\Gamma_{23}$ obtained by solving the Stokes problem by using the Lagrange multipliers but not including the coarse correction to the reduced spaces (so without guaranteeing the continuity and the physical correctness of stresses), then, as second option, including the coarse correction and not using the Lagrange 


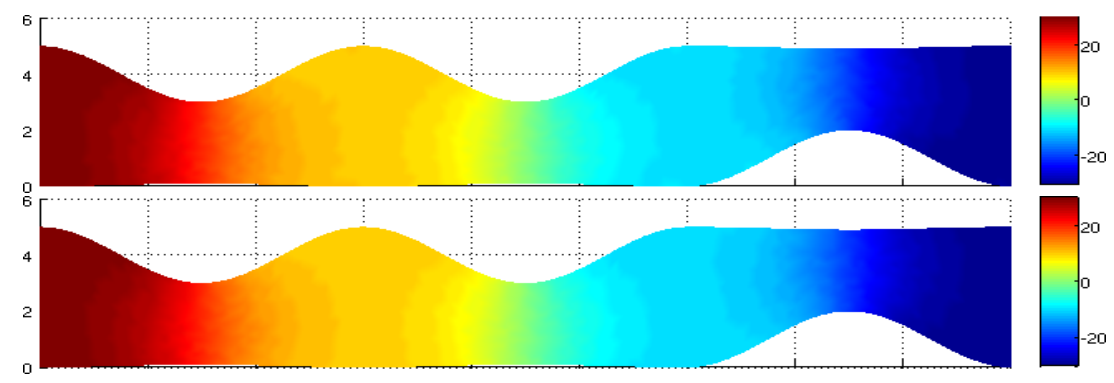

Figure 14: Pressure [ $\mathrm{Nm}^{-2}$ ] representative solutions using RBHM (with $N_{1}=N_{2}=N_{3}=$ 10) (top) and solving a global FEM (bottom), $\left(\boldsymbol{\mu}_{1}=(0.1,2), \boldsymbol{\mu}_{2}=(0.1,2), \boldsymbol{\mu}_{3}=(2,0.1)\right)$.

multipliers correction (not guaranteeing the continuity of velocity) and finally by using the RBHM method (that includes both velocity and coarse corrections). The profiles of the corresponding fine FEM solution computed in the whole network has been plotted in the same figure in order to compare the quality of the solutions.

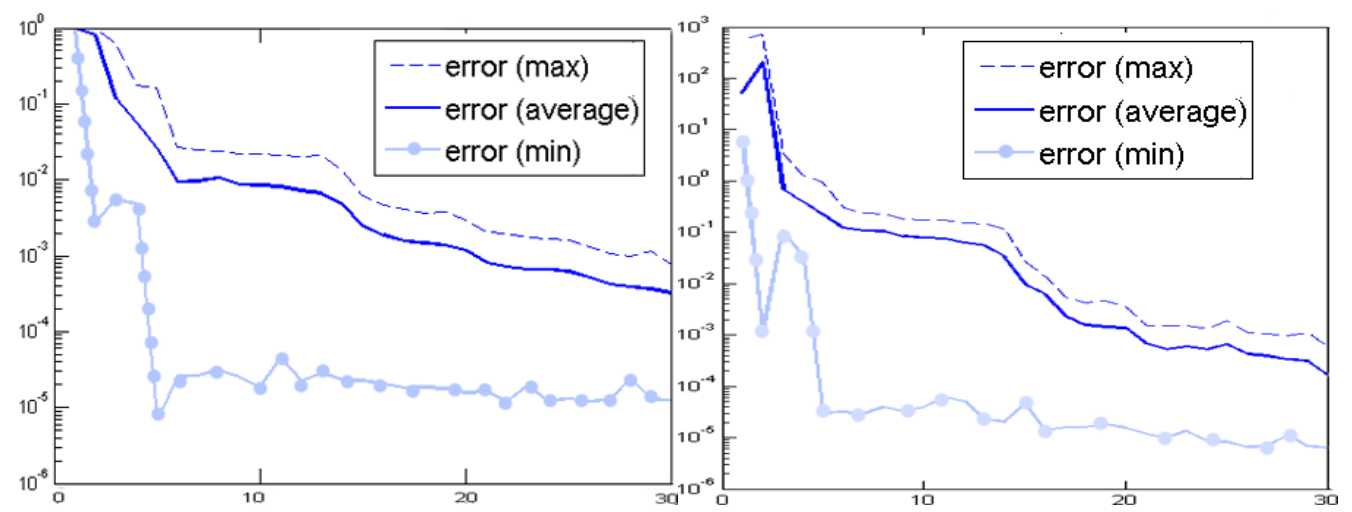

Figure 15: $H^{1}$ and $L^{2}$ relative error (minimum, maximum and average in a set of 1000 samples) on velocity (left) and pressure (right).

The same comparison on the velocity profiles is shown in Figure 18 and 19, while in Figures 20, 2122 and 23 is shown the comparison of the normal and tangential component profiles of the normal stress, defined respectively as $\boldsymbol{\sigma}_{n} \mathbf{n}=\nu \frac{\partial(\mathbf{u} \cdot \mathbf{n})}{\partial \mathbf{n}}-p$ and $\boldsymbol{\sigma}_{n} \mathbf{t}=\nu \frac{\partial(\mathbf{u} \cdot \mathbf{t})}{\partial \mathbf{n}}$.

In the figures shown, it is evident that the imposition of continuity of the velocities at the interfaces by Lagrange multipliers guarantees, by construction, the continuity of the tangential component of the normal stresses too, 


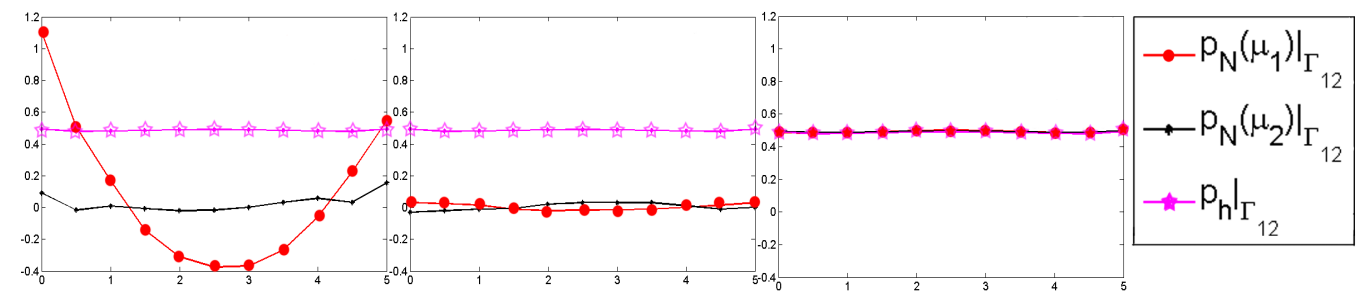

Figure 16: Pressure profiles along the internal interface $\Gamma_{12}$ without using the coarse correction (left), without using the Lagrange multipliers (center) and solving the complete RBHM problem (right).

while that of the normal component of the normal stresses is indeed satisfied thanks to the coarse correction.

The RBHM, by including the correction by Lagrange multipliers for the velocity and the coarse correction, provides an approximation of the solution that recovers the continuity of velocity and that of both components of normal stresses at the interfaces.

We remark that the use of Lagrange multipliers for velocity correction as proposed in RBEM is already able to guarantee the continuity of velocity with a good accuracy, lightly improved in the test with the use of RBHM and the continuity of the tangential component of normal stress, but not the continuity of the normal component of the normal stress.

Figure 24 shows the maximum error between the RBHM solution and the (true) FE fine solution. We note that when $H=h$, we add to the reduced space the couple $\left(\mathbf{u}_{H}, p_{H}\right)$ that is the "exact" solution of the interpolated problem. In this case this special basis will give all the contribution to the final solution and the error will be the smallest $\left(10^{-6}\right.$ in our case, since the empirical interpolation tolerance is set to this value). By increasing the value of $H$, the accuracy of the solution is given principally by that of the fine basis functions. Note however that, when increasing $H$, the error does not deteriorate. Otherwise said, the coarse solution guarantees the correct recovery of the physical normal stresses at the interfaces, without affecting the accuracy yielded by the reduced basis functions.

\subsection{A portion of a network with heterogeneous block domains}

In the second test case, the RBHM has been applied to the solution of the Stokes problem in a configuration that can be referred as a union of two 


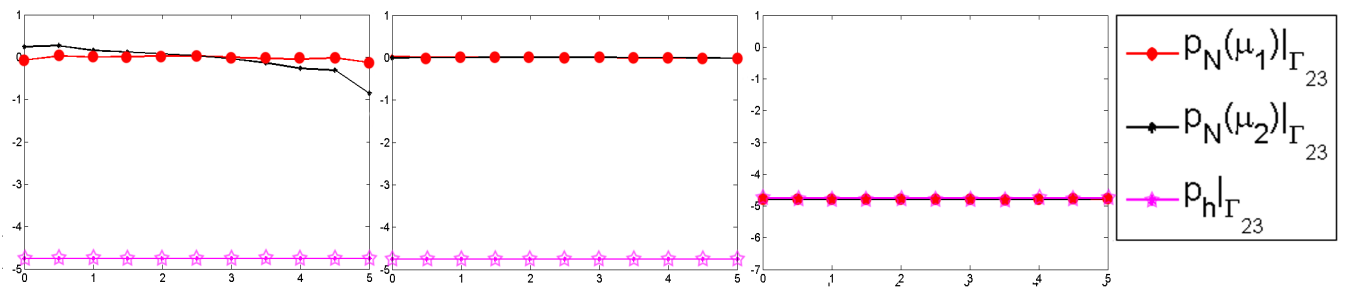

Figure 17: Pressure profiles along the internal interface $\Gamma_{23}$ without using the coarse correction (left), without using the Lagrange multipliers (center) and solving the complete RBHM problem (right).

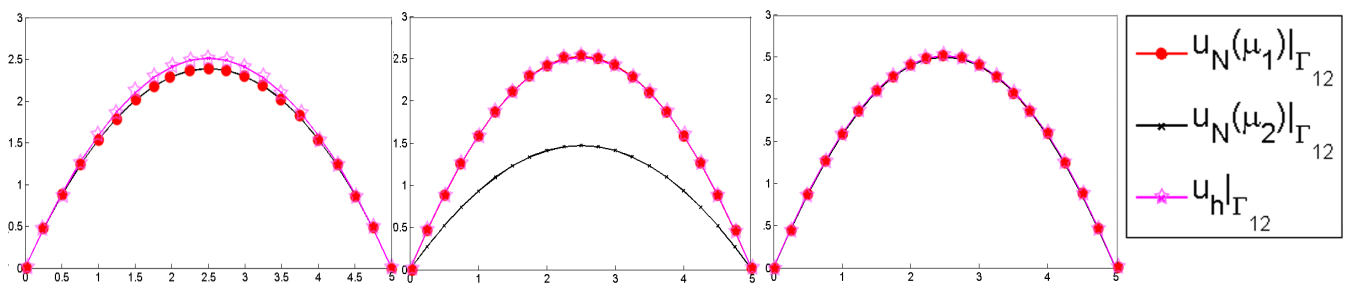

Figure 18: Velocity profiles along the internal interface $\Gamma_{12}$ without using the coarse correction (left), without using the Lagrange multipliers (center) and solving the complete RBHM problem (right).

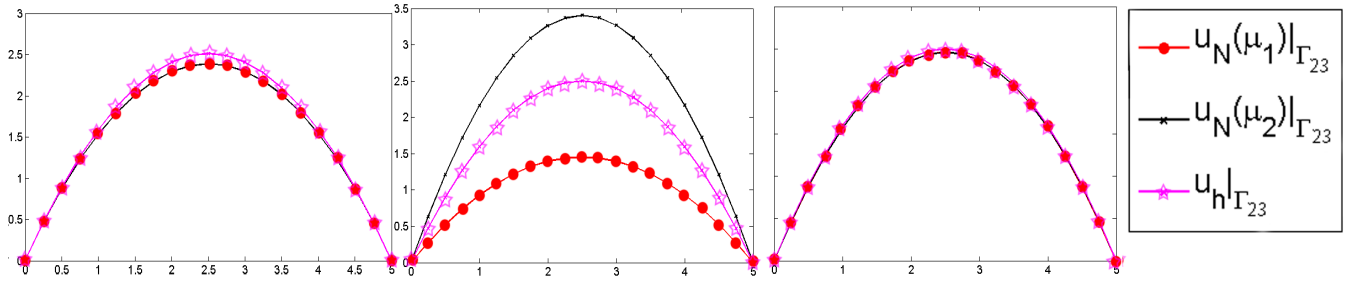

Figure 19: Velocity profiles along the internal interface $\Gamma_{23}$ without using the coarse correction (left), without using the Lagrange multipliers (center) and solving the complete RBHM problem (right).

subdomains, see Figure 25, that simulate respectively a bifurcation and a stenosis.

The two subdomains are obtained through two non-affine transfinite maps. The map that defines $\Omega_{1}$ depends on the bifurcation span $\mu_{1} \in[0.8,3.5]$. $\Omega_{2}$ is defined through the maps that depends on the parameter $\boldsymbol{\mu}_{2}=\left(\mu_{2}, \mu_{3}\right)$ that represents the amplitudes: $\mu_{2} \in[-1,1]$ and $\mu_{3} \in[-1,1]$, on the upper and lower walls representing the dilatation and the contraction of the pipe. This configuration can be used, for example, to model a carotid artery bifur- 


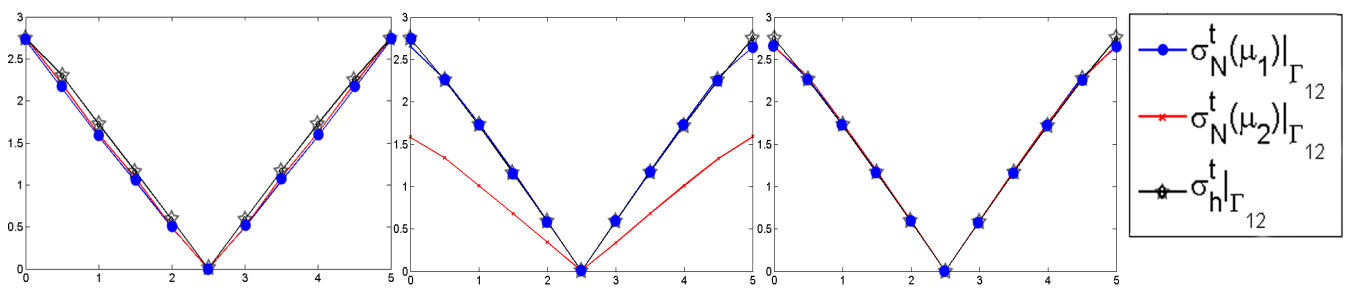

Figure 20: Tangential component of normal stress profiles along the internal interface $\Gamma_{12}$ without using the coarse correction (left), without using the Lagrange multipliers (center) and solving the complete RBHM problem (right).

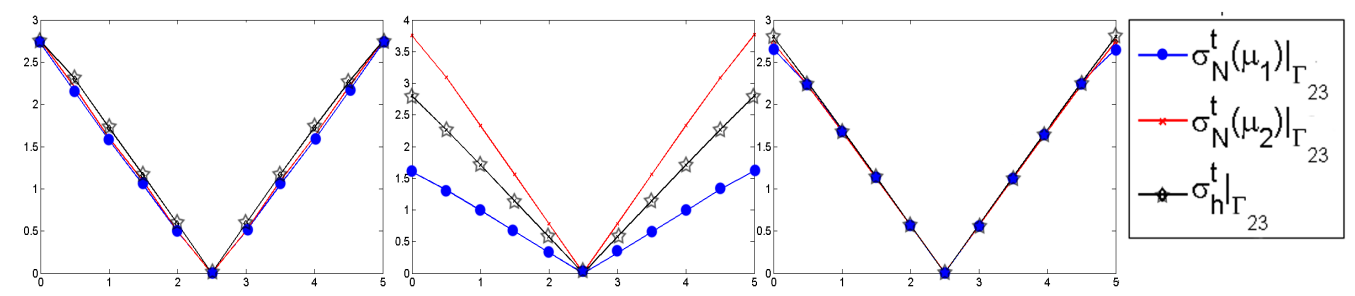

Figure 21: Tangential component of normal stress profiles along the internal interface $\Gamma_{23}$ without using the coarse correction (left), without using the Lagrange multipliers (center) and solving the complete RBHM problem (right).

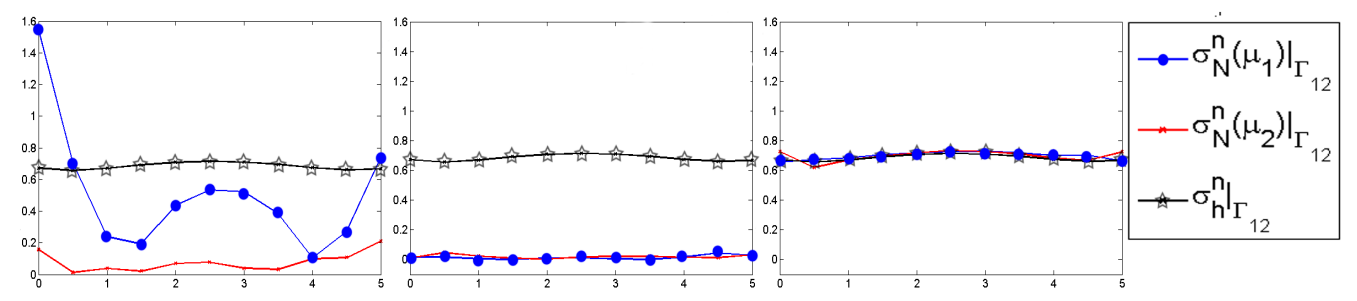

Figure 22: Normal component of normal stress profiles along the internal interface $\Gamma_{12}$ without using the coarse correction (left), without using the Lagrange multipliers (center) and solving the complete RBHM problem (right).

cation, [10].

We consider the bifurcation as the inflow subdomain in which we set homogeneous Dirichlet condition on the wall and on the lower branch of the bifurcation, Neumann boundary conditions given by imposing $\boldsymbol{\sigma}_{n}^{i n}=[10,0]^{T}$ on $\Gamma_{\text {in }}$ and $\boldsymbol{\sigma}_{n}^{\text {out }}=\mathbf{0}$ on the internal interface $\Gamma_{12}$. We assume that the stenosis block is the outflow domain and we set zero Dirichlet condition on the wall, while we impose Neumann boundary conditions $\boldsymbol{\sigma}_{n}^{i n}=\mathbf{0}$ on the internal interface $\Gamma_{12}$ and $\boldsymbol{\sigma}_{n}^{\text {out }}=[-10,0]^{T}$ on the outflow interface $\Gamma_{\text {out }}$. 


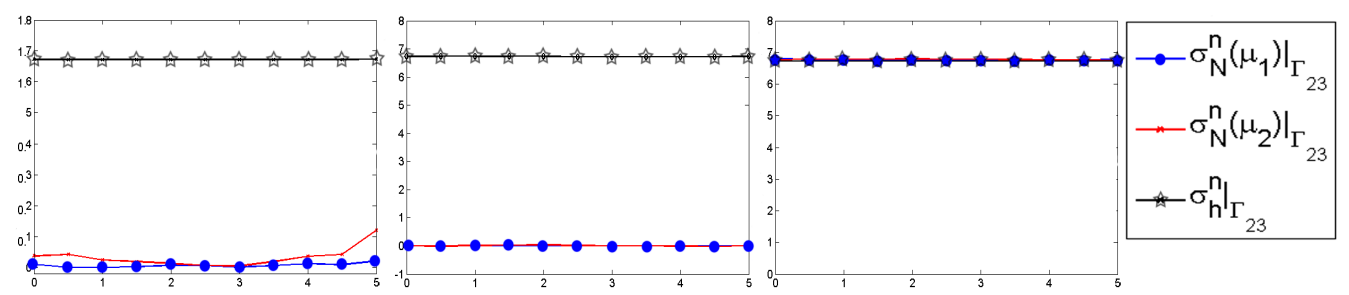

Figure 23: Normal component of normal stress profiles along the internal interface $\Gamma_{23}$ without using the coarse correction (left), without using the Lagrange multipliers (center) and solving the complete RBHM problem (right).

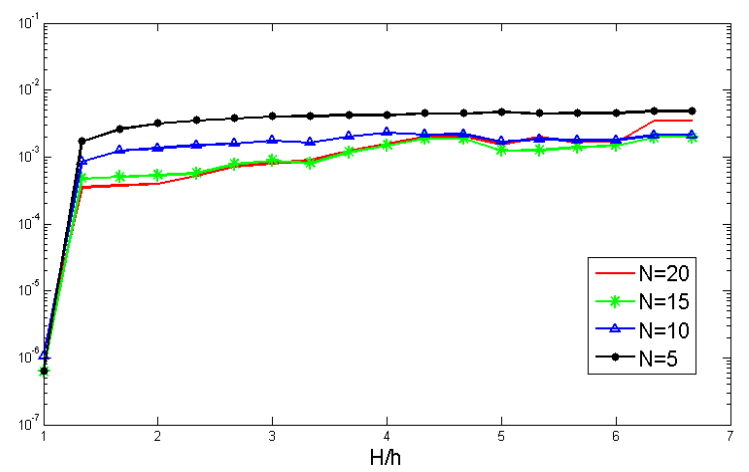

Figure 24: The effect of the coarse mesh on the overall accuracy of the RBHM.

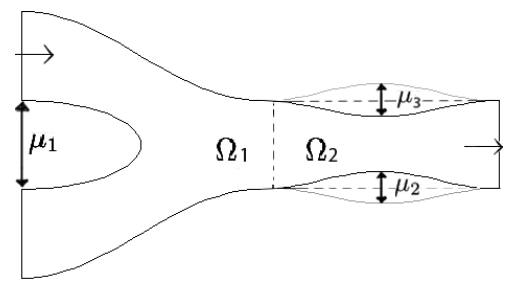

Figure 25: Geometrical scheme for the computational domain.

We apply the transfinite map to transform the problem in terms of reference coordinates.

By referring to a single block we expand each geometrical component in order to deal with an affine decomposition, as seen in Section 3. The terms (8), (11) and (12) are treated by the empirical interpolation method (EIM). The maximum interpolation error is set $\epsilon_{\text {tol }}^{E I M}=10^{-6}$. 
By applying the Greedy algorithm, we select $N_{1}=13$ parameters for $\boldsymbol{\mu}_{1}$ and $N_{2}=15$ parameter combinations for $\boldsymbol{\mu}_{2}=\left(\mu_{2}, \mu_{3}\right)$. Figure 26 shows the distribution of these parameters used to generate the basis functions.
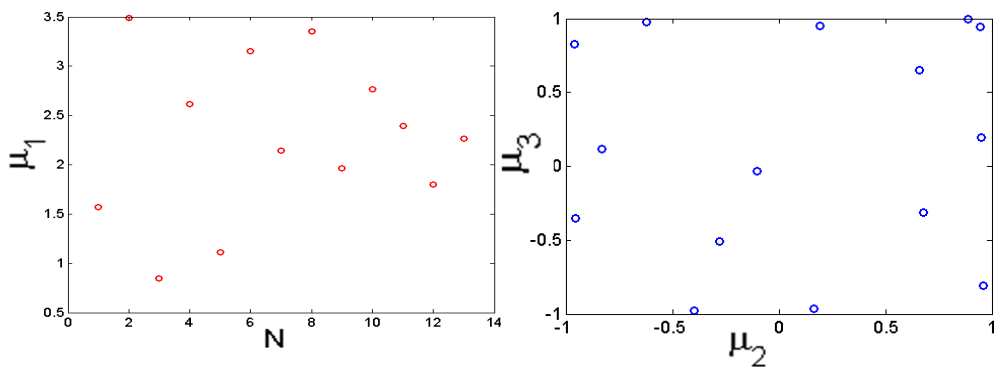

Figure 26: Parameter distribution representing the parameters combinations selected to generate the basis functions in the two blocks by greedy algorithm.

Coarse and fine grids have been chosen in order to deal with respectively 269 and 1006 nodes in the whole domain $\Omega$. Figures 27 and 28 show an example of flow solution, obtained using the reduced basis hybrid method, for a certain parameters combination $\left(\mu_{1}=2.5, \boldsymbol{\mu}_{2}=\left(\mu_{2}, \mu_{3}\right)=(1,0.9)\right)$, which can be compared with the solutions obtained with finite element method.
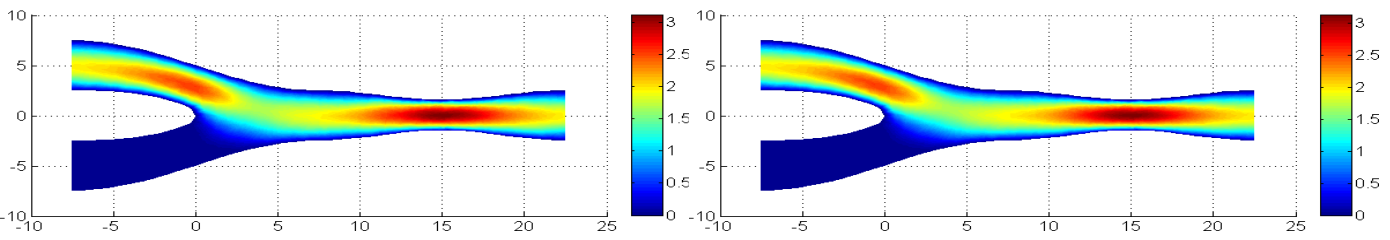

Figure 27: Velocity intensity $\left[\mathrm{ms}^{-1}\right]$ representative solutions using RBHM (with $N_{1}=$ $\left.N_{2}=10\right)$ (top) and by a global computed FEM solution (bottom), $\left(\mu_{1}=2.5, \boldsymbol{\mu}_{2}=\right.$ $(1,0.9))$.

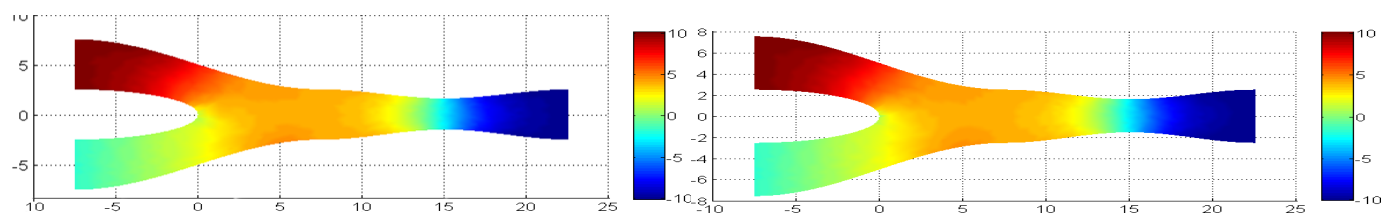

Figure 28: Pressure $\left[\mathrm{Nm}^{-2}\right]$ representative solutions using RBHM (with $N_{1}=N_{2}=10$ ) (top) and by a global computed FEM solution (bottom), $\left(\mu_{1}=2.5, \boldsymbol{\mu}_{2}=(1,0.9)\right)$. 


\section{Computational costs}

As already anticipated, the main feature of RBHM (as well as RBEM) is its capability to perform, thanks to the heavy computation done once in an offline stage, simulations on different combinations of the block domains by guaranteeing a certain versatility in combining several configurations and networks. The goal is to provide a method with lower complexity and lower computational times than the finite element method but able to guarantee an accurate solution and a certain physical reliability for velocity, pressure and stresses.

As already mentioned, the RBHM consists in two main steps. A parallel computational strategy can abate computational times, mainly in the offline step. If we have $K$ reference domains and we want to deal, for example, with "inflow", "central" and "outflow" elements, we can have a significant computational time reduction by computing the offline step with $3 \mathrm{~K}$ parallel processors, one for each reference element. During the online step, only the matrices assembling process can be partitioned in a parallel computation and, in this case, we need $\mathrm{R}$ processors, one for each subdomain. In Figure 29 the computational times required by the global finite elements solutions and by the online stage of the RBHM are represented by increasing the number of stenosis blocks of Section 7.1. The CPU time of RBHM breaks down into three components, respectively due to: the FEM coarse solution, the matrix assembling for each block, and the resolution of the reduced basis linear system. The second part can be computed independently for each block, on a parallel computational architecture where every processor deals with a single block.

The computational time of the online step of RBHM is not expensive and we have not big advantages by dealing with parallel computation in this step, nonetheless we have done it in order to show how every ingredient of the method contributes to the total computational time. In particular, in Figure 29 we can observe that if we treat each block by using parallel computation, the CPU time is mostly due to the FEM coarse solution. Thus we can obtain an online solution with an accuracy comparable with the one of the fine finite element solution at the cost of a coarse finite element solution. This achievement is gotten by reduced basis techniques and proper coupling conditions, where the coarse FEM solution is playing a crucial role (i.e. a lift) in guaranteeing the continuity of stresses. We also underline that the computational advantages are more evident for extended networks and an 
increasing number of blocks.

In order to visualize the different computational loads and the advantage of the reduced model proposed, we report in Table 1 the values of the CPU times in details for different number $R$ of subdomains, the computational time for the matrix assembling is $0.76 \mathrm{~s}$ for each of the subdomains considered. The last column underlines the computational costs of RBHM compared with the fine FEM solution. We can see that, in terms of computational cost, the fine solution computed with RBHM is comparable to the one obtained on the coarser grid with standard FEM, and in general the computational savings are of two orders of magnitude with RBHM compared with FEM. Moreover the CPU time spent for a FEM simulation with 5 blocks is on par with RBHM simulation dealing with a domain defined by 27 blocks, still retaining the continuity of velocities and stresses at the interfaces.

\begin{tabular}{c|ccccc}
\hline \hline R & $\begin{array}{c}\text { Fine FEM } \\
\text { solution }\end{array}$ & $\begin{array}{c}\text { Coarse FEM } \\
\text { solution }\end{array}$ & $\begin{array}{c}\text { Reduced Linear } \\
\text { System }\end{array}$ & $\begin{array}{c}\text { RBHM } \\
\text { solution }\end{array}$ & $\begin{array}{c}\text { RBHM vs } \\
\text { Fine FEM(\%) }\end{array}$ \\
\hline 5 & 31.13 & 1.73 & 0.06 & 2.72 & 8.76 \\
10 & 132.18 & 4.86 & 0.14 & 5.68 & 4.30 \\
15 & 311.44 & 10.18 & 0.23 & 11.08 & 3.56 \\
20 & 557.57 & 16.77 & 0.28 & 17.81 & 3.19 \\
25 & 880.54 & 23.86 & 0.60 & 25.22 & 2.86 \\
30 & 1183.5 & 34.81 & 0.78 & 36.35 & 3.07 \\
35 & 1895.7 & 49.74 & 1.02 & 51.52 & 2.71 \\
40 & 2484.6 & 70.44 & 1.56 & 72.76 & 2.92 \\
\hline
\end{tabular}

Table 1: Computational times (in seconds) of FEM and RBHM for different number of subdomains R.

\section{Comparison with classical Reduced Basis Method (RBM)}

The classical reduced basis method is used when we want to solve rapidly a large number of problems governed by the same partial differential equation that depends on parameters $[22 ; 32 ; 36]$. It has been developed in a mono-domain case and it is highly efficient when we deal with geometry endowed with topological similarities $[22 ; 23]$. If we want to consider repetitive and heterogeneous geometries composing a network, an offline computation for each new combination of domain configuration has to be performed. The reduced basis element method RBEM avoids this problem and allows dealing 


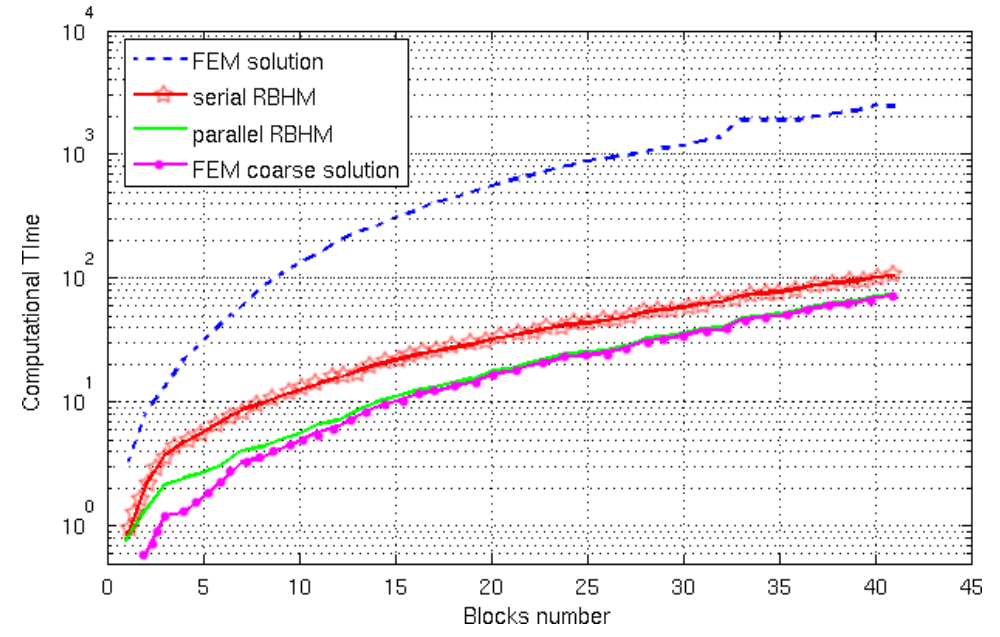

Figure 29: Computational times (in seconds) of FEM and RBHM

with every kind of combinations of a certain number of blocks, for which few offline stages can be computed independently. The proposed hybrid version, RBHM, combines the previous approach with a FEM coarse solution to guarantee the continuity of both velocity and stresses solutions across interfaces. We want now to consider a three stenoses configuration for a comparative analysis between the reduced basis method (RBM) and the reduced basis hybrid method (RBHM). Using RBM we have to perform the offline stage in the whole domain characterized by six parameters (two for each stenoses), coupling conditions are automatically satisfied. During this step, the empirical interpolation generates 90 terms for the affine decomposition of the bilinear forms and the greedy algorithm needs 49 basis functions in order to reach an error with $10^{-4}$ of tolerance.

Using the proposed RBHM we compute the offline stage in a single stenosed domain, we consider smaller number of parameters (only two) allowing to deal with just 29 terms for the affine decomposition of the bilinear forms representing the problems (always carried out by the empirical interpolation) and 14 basis functions to reach an error of order $10^{-3}$. The comparison between the features of the two methods is shown in Table 2 . The complexity reduction is very important also for the offline step.

Figure 30 shows how the number of parameters affects the choice of basis functions. We can observe that, during the greedy RB spaces assembling, in the case of three stenosed domains (6 parameters), we need more than 
three times the number of basis functions compared with the number we need in the case of a single stenosis in order to reach the same convergence relative error (2 parameters). In the single domain case, for a tolerance on the greedy algorithm of $\epsilon=10^{-3}$ we need just $N=7$, in the three stenosis domain $N=26$, while for $\epsilon=10^{-4}$, respectively, $N=11$ and $N=45$. In both cases we can conclude that three times the number of basis for the single domain case is less than the number of basis that we have in the three stenosis domain $^{4}, 3 N=21<26$ and $3 N=33<45$. With the application of RBHM we can reduce also the complexity of the operators representing the problem ( $M^{k a}$ and $M^{k b}$ of (14) and (15) ) and the dimension of the RB spaces. This is useful also for a good performance of a posteriori error bounds $[33]$.

\begin{tabular}{c|cc}
\hline \hline & RBM & RBHM \\
\hline$H^{1}$ rel. vel. error & $1 \mathrm{E}-04$ & $1 \mathrm{E}-04$ \\
$\mathrm{~N}$ & 45 & $3 \times 14$ \\
$\epsilon_{E I M}$ & $1 \mathrm{E}-06$ & $1 \mathrm{E}-06$ \\
$M^{k a}$ & 71 & 24 \\
$M^{k b}$ & 19 & 5 \\
\hline
\end{tabular}

Table 2: Computational complexity of RBM and RBHM.

\section{Numerical test on a $3 \mathrm{D}$ domain}

In this section we apply the RBHM to 3D domain in order to address more realistic configurations for blood flow in the study of stenosed arteries. Here, Taylor-Hood Finite Element Method has been used to compute approximation basis functions, $\mathbb{P}_{2}$ elements for velocity and supremizer, $\mathbb{P}_{1}$ for pressure, respectively [30] and consequently $\mathbb{P}_{1}\left(\Gamma_{l m}\right)$ for the Lagrange multipliers space.

The RBHM has been applied to solve the Stokes equations in a computational domain $\Omega$ composed by two stenosed blocks $\Omega_{\mu_{1}}$ and $\Omega_{\mu_{2}}$ (Figure 31).

\footnotetext{
${ }^{4}$ Of course we have to take into account the further costs and effort of the coupling condition in the use of RBHM, but the proposed method still keeps reasonable computational advantages.
} 


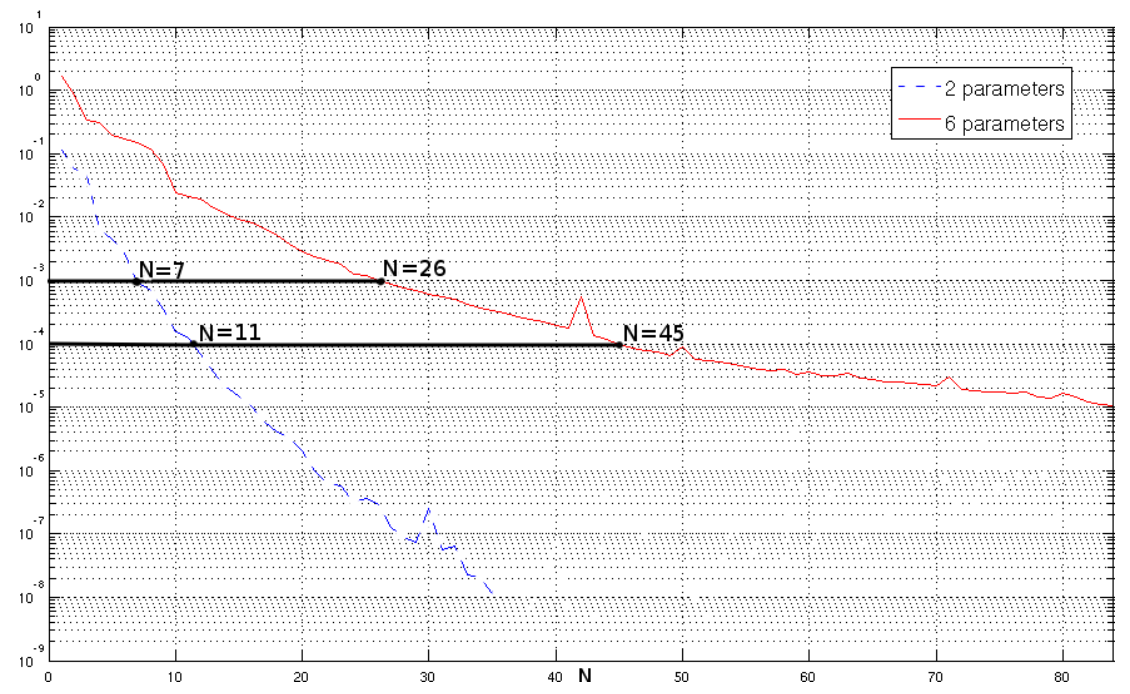

Figure 30: Relative $H^{1}$ velocity errors during the greedy RB spaces assembling dealing with 2 parameters and 6 parameters.

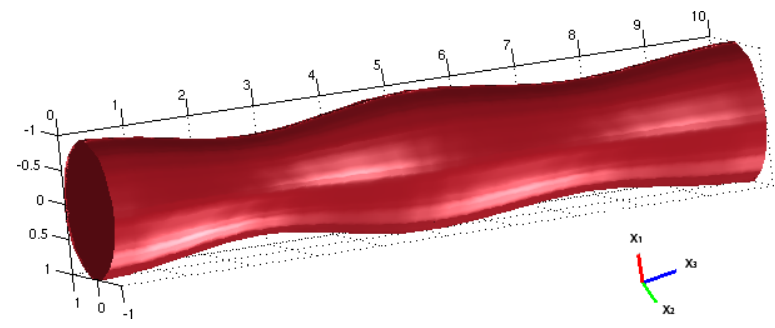

Figure 31: Computational domain $\left.\mu_{1}=7, \mu_{2}=10\right)$.

The geometry of a single stenosis is obtained by the deformation of a reference pipe through a parameter that represents the contraction in the middle of the pipe.

The deformed domain $\Omega_{\mu}$ is mapped from the straight reference pipe $\hat{\Omega}$ of length $L=5$ and radius $r=1$ through the following coordinate transforma- 
tion $T_{\mu}: \hat{\Omega} \rightarrow \Omega_{\mu}$ such as $\mathbf{x}=T_{\mu}(\hat{\mathbf{x}})$ and

$$
\begin{aligned}
& x_{1}=\hat{x}_{1}+\frac{\hat{x}_{1}}{\mu}\left(\cos \left(\frac{2 \pi \hat{x}_{3}}{L}\right)-1\right) \\
& x_{2}=\hat{x}_{2}+\frac{\hat{x}_{2}}{\mu}\left(\cos \left(\frac{2 \pi \hat{x}_{3}}{L}\right)-1\right) \\
& x_{3}=\hat{x}_{3}
\end{aligned}
$$

The range of the parameter $\mu$ is $[-20,-5] \cup[5,20]$, Figure 32 shows the reference pipe and some representative deformations of the arterial portion after the deformation.

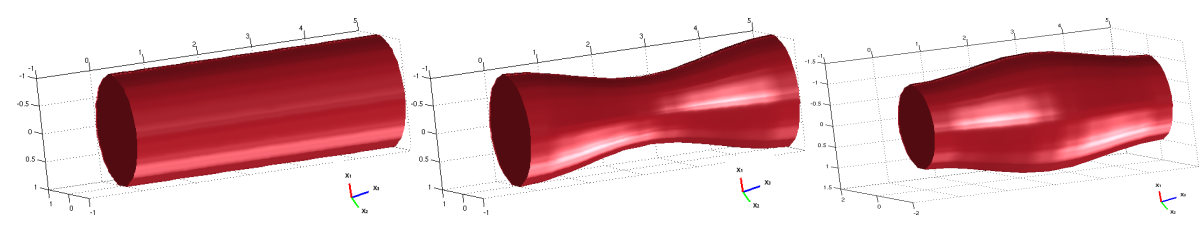

Figure 32: Reference pipe and two deformed pipes $(\mu=5, \mu=-5, \mu=5)$ : stenosis and aneurysm configuration.

We consider a parametrized Stokes problem for each subdomain. For the inflow subdomain, we compute the reduced basis imposing zero Dirichlet condition on the wall, Neumann boundary conditions given by imposing $\boldsymbol{\sigma}_{n}=\boldsymbol{\sigma} \cdot \mathbf{n}=\nu \frac{\partial \mathbf{u}}{\partial \mathbf{n}}-p \mathbf{n}$ to be $\boldsymbol{\sigma}_{n}^{i n}=[0,0,5]^{T}$ on $\Gamma_{i n}$ and $\boldsymbol{\sigma}_{n}^{\text {out }}=\mathbf{0}$ on the internal interface $\Gamma_{12}$. For the outflow subdomain, we compute the reduced basis imposing zero Dirichlet condition on the wall, Neumann boundary conditions imposing $\boldsymbol{\sigma}_{n}^{\text {in }}=\mathbf{0}$ on the internal interface $\Gamma_{23}$ and $\boldsymbol{\sigma}_{n}^{\text {out }}=[0,0,-1]^{T}$ on the outflow interface $\Gamma_{\text {out }}$. Figure 33 shows the distribution of the parameter values selected by the greedy algorithm, by applying the offline stage of the reduced basis method to the single stenosis block. By taking into account that the range $[-5,5]$ is not admitted, we can see that the higher concentration of values is in the intervals $[-10,-5]$ and $[5,10]$ in correspondence of larger deformation of the pipe.

Coarse and fine grids have been chosen in order to deal with respectively 155 and 2714 nodes in a single block domain. Figure 34 shows a representative flow solution in $\Omega$, found with the reduced basis hybrid method, to be com- 


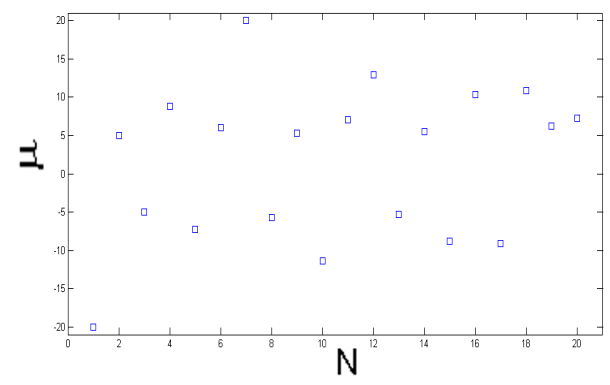

Figure 33: Distribution of the selected parameter values by the greedy algorithm used to generate the basis functions in a single block.

pared with the finite element solution. The same comparison, regarding the pressure solutions, is shown in Figure 35.

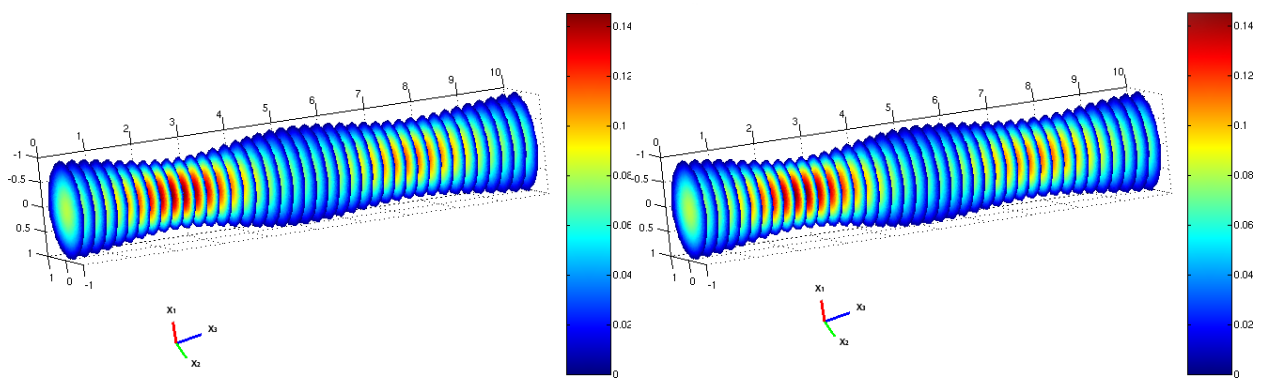

Figure 34: Representative solutions of velocity $\left[\mathrm{ms}^{-1}\right]$ using RBHM (with $N_{1}=N_{2}=19$ ) (left) and using FEM as a global solution (right), $\mu_{1}=7, \mu_{2}=10$.

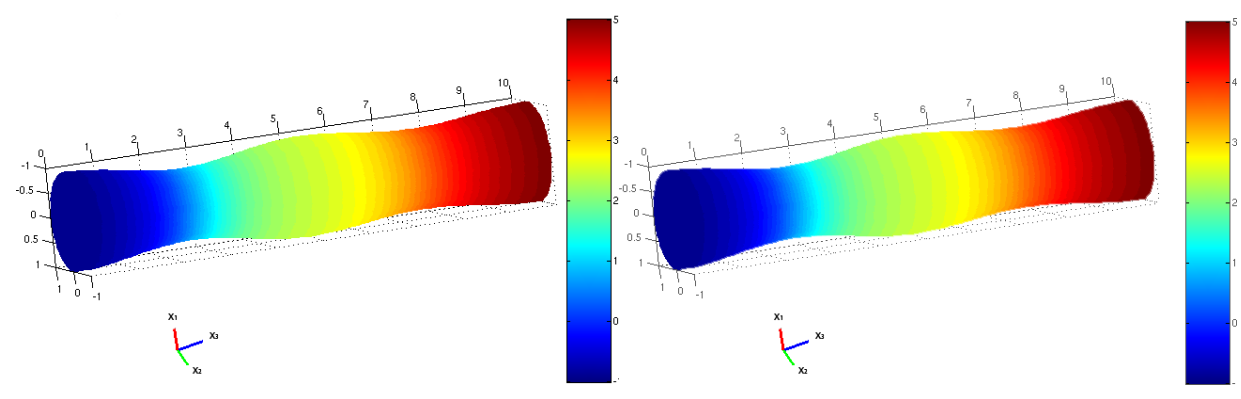

Figure 35: Representative solutions pressure $\left[\mathrm{Nm}^{-2}\right]$ using RBHM (with $N_{1}=N_{2}=19$ ) (left) and using FEM as a global solution (right), $\mu_{1}=7, \mu_{2}=10$.

Figure 36 shows the reduction of the $H^{1}$ relative errors on velocity and $L^{2}$ relative errors on pressure, respectively, for the configuration of Figures 34 and 35 , versus the number $\mathrm{N}$ of basis functions. 


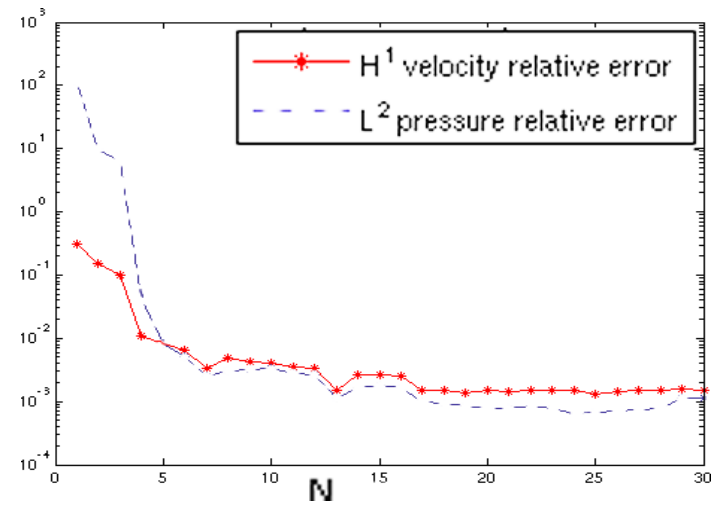

Figure 36: $H^{1}$ and $L^{2}$ relative errors on velocity and pressure.

As in the 2D case, we show in Figure 37 a comparison regarding the velocity profiles on the internal interface obtained by using the RBHM (that includes both velocity and coarse corrections). The profiles of the correspondig fine FEM global solution has been plotted in order to compare the quality of the solution. Figure 38 shows the velocity profiles on the internal interface $\Gamma_{12}$ obtained solving the Stokes problem by using the Lagrange multipliers but not including the coarse correction to the reduced spaces (so without guaranteeing the continuity of stresses). Figure 39, shows the velocity profiles on the internal interface $\Gamma_{12}$ obtained including the coarse correction and not using the Lagrange multipliers correction (not guaranteeing the continuity of velocity). The profiles of the corresponding fine FEM solution computed in the whole network has been plotted as well in order to compare the quality of the solutions. The solutions on $\Omega$ for both options are shown in Figures 40 and 41 in order to compare the pressure as well.

The reduced basis hybrid method RBHM allows dealing with every kind of combinations of a certain number of blocks, for which few offline stages can be computed independently. Using RBM we have to perform the offline stage in the whole domain characterized by two parameters, coupling conditions are automatically satisfied. Using the proposed RBHM, the greedy algorithm is computed during the offline stage in a single stenosed domain.

Figures 30 and 42 report the true errors and show how the number of parameters affects the choice of basis functions. We can observe that, during the greedy RB spaces assembling, in the case of two stenosed domains (2 parameters), we need more than two times the number of basis functions compared with the number we need in the case of a single stenosis in order to reach the 

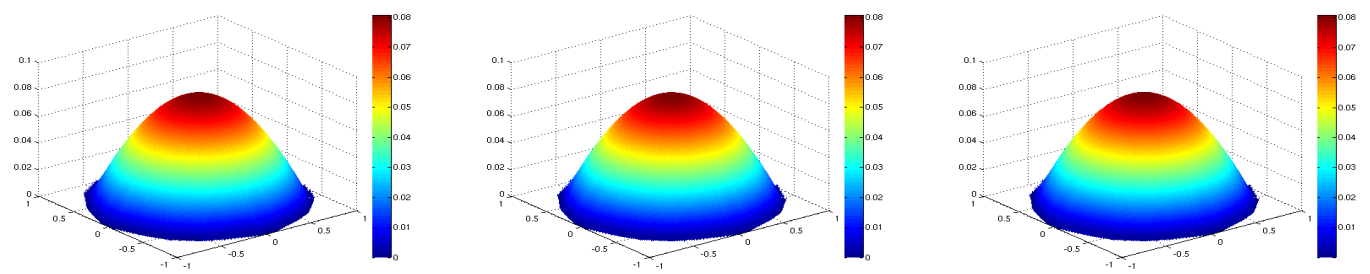

Figure 37: Velocity profiles along the internal interface $\Gamma_{12}$ by solving the RBHM problem plotted from the first block (left), from the second block (center), compared with the velocity profile obtained by using the global FEM solution along the same internal interface (right).
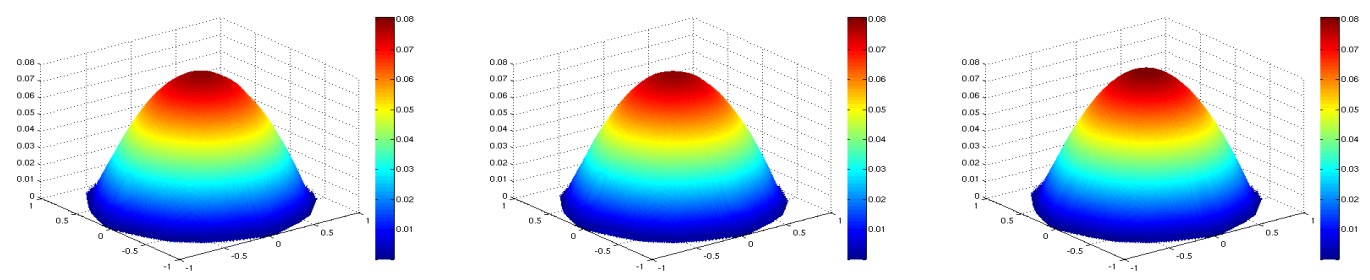

Figure 38: Velocity profiles along the internal interface $\Gamma_{12}$ by using the velocity correction and not using the coarse correction, plotted from the first block (left), from the second block (center), compared with the velocity profile obtained by using the global FEM solution along the same internal interface (right).
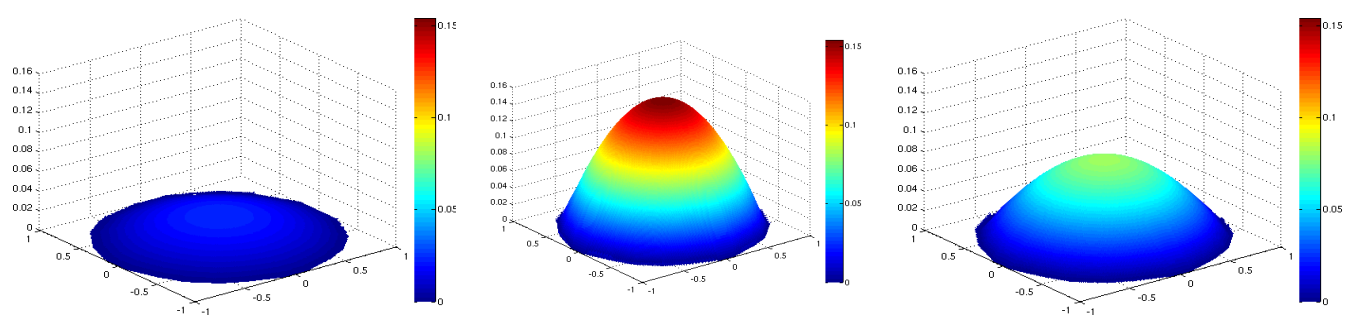

Figure 39: Velocity profiles along the internal interface $\Gamma_{12}$ by not using the velocity correction and including the coarse correction, plotted from the first block (left), from the second block (center), compared with the velocity profile obtained by using the global FEM solution along the same internal interface (right).

same convergence relative error (1 parameter). In the single domain case, for a tolerance on the greedy algorithm of $\epsilon=10^{-7}$ we need just $N=9$, in the two stenosed domain $N=22$, while for $\epsilon=10^{-11}$, respectively, $N=12$ and $N=45$. In both cases we can conclude that two times the number of basis 

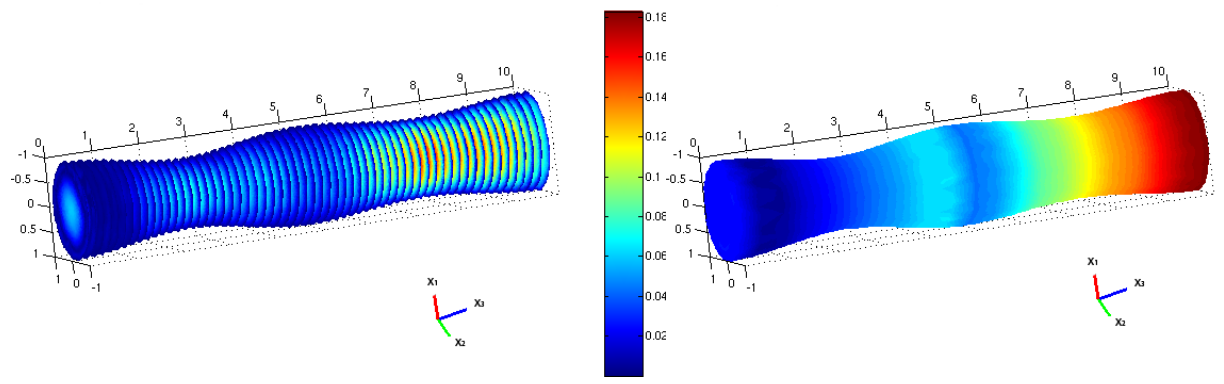

Figure 40: Representative solutions of velocity $\left[\mathrm{ms}^{-1}\right]$ and pressure $\left[\mathrm{Nm}^{-2}\right]$ using only the velocity correction but not using the coarse correction (with $N_{1}=N_{2}=19$ ), $\mu_{1}=$ $7, \mu_{2}=10$.

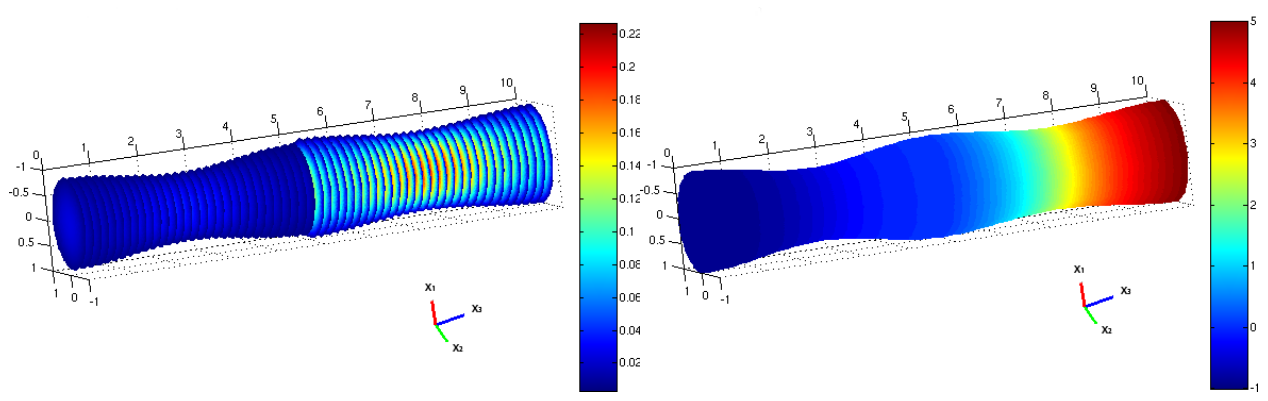

Figure 41: Representative solutions of velocity $\left[\mathrm{ms}^{-1}\right]$ and pressure $\left[\mathrm{Nm}^{-2}\right]$ using the coarse correction but not the velocity correction (with $N_{1}=N_{2}=19$ ), $\mu_{1}=7, \mu_{2}=10$.

for the single domain case (that we need for RBHM) is less than the number of basis that we have in the two stenosis domain (that we need for RBM) ${ }^{5}$, $2 N=18<22$ and $2 N=24<45$. With the application of RBHM we can reduce efficiently also the complexity and the dimension of the RB spaces.

\footnotetext{
${ }^{5}$ Of course we have to take into account the further costs and effort of the coupling condition in the use of RBHM, but the proposed method still keeps computational advantages and gives continuous pressure and stresses.
} 


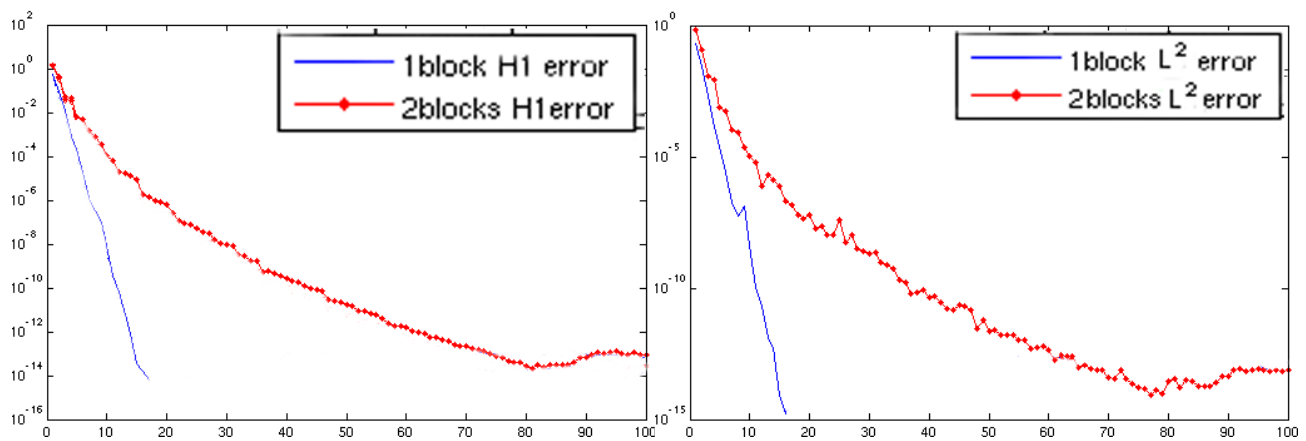

Figure 42: Relative $H^{1}$ velocity errors (left) and $L^{2}$ pressure errors (right) during the greedy RB spaces assembling dealing with 1 block and 2 blocks.

\section{Conclusions and perspectives}

In this work we have proposed an extension of the reduced basis element method [17] in a multi-domain flow network by introducing a reduced basis hybrid method (RBHM). The latter allows solving the fluid flow problems in more complex geometries, to deal with a computational domain decomposed by several combinations of repetitive blocks on which the solution can be computed locally and quickly thanks to the classical RB method, and then properly coupled and glued guaranteeing the continuity of velocity and stresses at the subdomains interfaces.

The geometrical deformations are computed through a non-affine transfinite map and an empirical interpolation method has been used to perform a complete offline/online computational decoupling of the reduced basis problem. Results dealing with the complexity reduction and computational performances have been provided in comparison with classical finite element techniques and classical reduced basis method on two test cases of interest.

Future developments will include geometrical deformations to apply the methodology in a more complex three-dimensional setting, such that we may increase the geometrical complexity. In particular the reduced basis hybrid method will be exploited for nonlinear Navier-Stokes equations in parametrized domains. The interest is to apply this methodology to three-dimensional relevant configurations in cardiovascular problems in an efficient, accurate and real-time framework by keeping all the previous advances in the development of reduced basis method [23]. 


\section{Acknowledgements}

This work has been supported by the Swiss National Science Foundation

under the Project 122136 and by ERC-Mathcard Project (ERC-2008-AdG2270058).

\section{References}

[1] V. Agoshkov, A. Quarteroni, and G. Rozza. Shape design in aortocoronaric bypass anastomoses using perturbation theory. SIAM J. Numer. Anal., 44(1):367-384, 2006.

[2] D. Ambrosi, A. Quarteroni, and G. Rozza. Modelling of Physiological Flows. Springer, Series MS\&A, Vol. 5, 2011.

[3] M. Barrault, Y. Maday, N.C. Nguyen, and A.T. Patera. An "empirical interpolation" method: application to efficient reduced-basis discretization of partial differential equations. C.R Acad. Sci. Paris Anal. Numerique 339, pages 667-672, 2004.

[4] F. Brezzi and M. Fortin. Mixed and Hybrid Finite Element Methods. Springer Verlag, 1991.

[5] C. Canuto, M.Y. Hussaini, and T. A. Quarteroni, A. Zang. Spectral Methods: Evolution to Complex Geometries and Applications to Fluid Dynamics. Springer, 2007.

[6] Y. Chen, J.S. Hesthaven, and Y. Maday. A Seamless Reduced Basis Element Method for 2D Maxwell's Problem: An Introduction. In J. S. Hesthaven and E. M. Rønquist, editors, Spectral and High Order Methods for Partial Differential Equations, volume 76, pages 141-152. Springer Berlin Heidelberg, 2011.

[7] S. Deparis. Reduced basis error bound computation of parameterdependent Navier-Stokes equations by the natural norm approach. SIAM J. Numer. Anal., 46(4):2039-2067, 2008.

[8] S. Deparis and E. Løvgren. Stabilized reduced basis approximation of incompressible three-dimensional Navier-Stokes equations in parametrized deformed domains. Journal of Scientific Computing, pages 1-15, 2011. 
[9] S. Deparis and G. Rozza. Reduced basis method for multi-parameterdependent steady Navier-Stokes equations: Applications to natural convection in a cavity. Journal of Computational Physics, 228(12):43594378, 2009.

[10] L. Formaggia, A. Quarteroni, and A. Veneziani. Cardiovascular Mathematics. Modeling and simulation of the circulatory system. Springer, Series MS\&A, Vol. 1, 2009.

[11] W. Gordon and C. Hall. Transfinite element method: blending-function interpolation over arbitrary curved element domains. Numerische Mathematik, 21:109-129, 1973.

[12] D.B.P. Huynh, D.J. Knezevic, and A.T. Patera. A static condensation reduced basis element method: Approximation and a posteriori error estimation. Submitted to Mathematical Modelling and Numerical Analysis, 2011.

[13] D.J. Knezevic, N.C. Nguyen, and A.T. Patera. Reduced basis approximation and a posteriori error estimation for the parametrized unsteady Boussinesq equations. Mathematical Models and Methods in Applied Sciences, 2010.

[14] T. Lassila and G. Rozza. Parametric free-form shape design with pde models and reduced basis method. Computer Methods in Applied Mechanics and Engineering, 199(23-24):1583-1592, 2010.

[15] A. E. Løvgren, Y. Maday, and E. M. Rønquist. The reduced basis element method: Offline-online decomposition in the nonconforming, nonaffine case. In J. S. Hesthaven and E. M. Rønquist, editors, Spectral and High Order Methods for Partial Differential Equations, volume 76, pages 247-254. Springer Berlin Heidelberg, 2011.

[16] A.E. Løvgren, Y. Maday, and E.M. Rønquist. A reduced basis element method for the steady Stokes problem. Mathematical Modelling and Numerical Analysis, 40(3):529-552, 2006.

[17] A.E. Løvgren, Y. Maday, and E.M. Rønquist. A reduced basis element method for complex flow systems. Proceedings of ECCOMAS CFD, P. Wesseling, E. Onate, J. Periaux (Eds.) TU Delft, The Netherlands, 2006. 
[18] A.E. Løvgren, Y. Maday, and E.M. Rønquist. Global $\mathrm{C}^{1}$ maps on general domains. Mathematical Models and Methods in Applied Sciences (M3AS), 19(5):803-832, 2009.

[19] A.E. Løvgren, Y. Maday, and E.M. Rønquist. The spectral element method used to assess the quality of a global $\mathrm{C}^{1}$ map. In J. S. Hesthaven and E. M. Rønquist, editors, Spectral and High Order Methods for Partial Differential Equations, volume 76, pages 441-448. Springer Berlin Heidelberg, 2011.

[20] Y. Maday and E.M. Rønquist. A reduced-basis element method. J.Sci. Comput., 17:447-459, 2002.

[21] Y. Maday and E.M. Rønquist. The reduced-basis element method: Application to a thermal fin problem. SIAM J.Sci. Comput., 26:240-258, 2004.

[22] A. Manzoni, A Quarteroni, and G. Rozza. Shape optimization for viscous flows by reduced basis methods and free-form deformation techniques. In press.

[23] A. Manzoni, A. Quarteroni, and G. Rozza. Model reduction techniques for fast blood flow simulation in parametrized geometries. International Journal for Numerical Methods in Biomedical Engineering, 2011. In press.

[24] N.C. Nguyen, K. Veroy, and A.T. Patera. Certified real-time solution of parametrized partial differential equations. Handbook of Materials Modeling, S. Yip Ed., Kluwer Academic Publishing, Springer, 2005.

[25] A.T. Patera and G. Rozza. Reduced Basis Approximation and A Posteriori Error Estimation for Parametrized Partial Differential Equations. Version 1.0, Copyright MIT 2006, to appear in (tentative rubric) MIT Pappalardo Graduate Monographs in Mechanical Engineering. Available at http://augustine.mit.edu.

[26] A. Quarteroni. Numerical Models for Differential Problems. Springer, Series MS\&A, Vol. 2, 2008. 
[27] A. Quarteroni and G. Rozza. Optimal control and shape optimization of aorto-coronaric bypass anastomoses. Math. Models Meth. Appl. Sci., 13(12):1801-1823, 2003.

[28] A. Quarteroni and G. Rozza. Numerical solution of parametrized NavierStokes equations by reduced basis methods. Numer. Methods Partial Differential Equations, 23(4):923-948, 2007.

[29] A. Quarteroni and A. Valli. Numerical Approximation of Partial Differential Equations. Springer-Verlag, 1994.

[30] A. Quarteroni and A. Valli. Domain Decomposition Methods for Partial Differential Equations. Oxford University Press, Oxford, 1999.

[31] G. Rozza. Shape design by optimal flow control and reduced basis techniques: applications to bypass configurations in haemodynamics. $\mathrm{PhD}$ thesis, École Polytechnique Fédérale de Lausanne, 2005. N. 3400, http://infoscience.epfl.ch.

[32] G. Rozza. Reduced basis methods for Stokes equations in domains with non-affine parameter dependence. Comput. Vis. Sci., 12(1):23-35, 2009.

[33] G. Rozza, D.B.P. Huynh, and A. Manzoni. Reduced basis approximation and a posteriori error estimation for stokes flows in parametrized geometries: roles of the inf-sup stability constants. Submitted, 2010.

[34] G. Rozza, D.B.P. Huynh, and A.T. Patera. Reduced basis approximation and a posteriori error estimation for affinely parametrized elliptic coercive partial differential equations. Arch. Comput. Methods Engrg., 15:229-275, 2008.

[35] G. Rozza, C.N. Nguyen, A.T. Patera, and S. Deparis. Reduced basis methods and a posteriori error estimators for heat transfer problems. Proceedings of HT2009, ASME Summer Heat Transfer Conference, San Francisco, CA, USA, paper HT 2009-88211, 2:753-762, 2009.

[36] G. Rozza and K. Veroy. On the stability of the reduced basis method for Stokes equations in parametrized domains. Comput. Meth. Appl. Mech. Engr., 196(7):1244-1260, 2007. 
[37] K. Veroy and A.T. Patera. Certified real-time solution of the parametrized steady incompressible Navier-Stokes equations: rigorous reduced-basis a posteriori error bounds. International Journal for $\mathrm{Nu}$ merical Methods in Fluids, 47 (8-9):773-788, 2005. 